\title{
A Necroptosis-Related Gene Signature for Predicting Prognosis, Immune Landscape, and Therapy Sensitivity in Hepatocellular Carcinoma
}

\author{
Junliang Chen
}

Shengjing Hospital of China Medical University

Huaitao Wang

Shengjing Hospital of China Medical University

Lei Zhou

Shengjing Hospital of China Medical University

Zhihao Liu

Shengjing Hospital of China Medical University

\section{Hui Chen}

Shengjing Hospital of China Medical University

Xiaodong Tan ( $\nabla$ tanxd@sj-hospital.org )

Shengjing Hospital of China Medical University

\section{Research Article}

Keywords: Hepatocellular carcinoma, Necroptosis, TCGA, ICGC, GEO, Prognosis, Gene signature, Nomogram, Therapy

Posted Date: January 3rd, 2022

DOI: https://doi.org/10.21203/rs.3.rs-1201594/v1

License: (c) (1) This work is licensed under a Creative Commons Attribution 4.0 International License. Read Full License 


\section{Abstract}

Background: Hepatocellular carcinoma (HCC) remains a growing threat to global health. Necroptosis is a newly discovered regulated cell necrosis that plays a vital role in cancer development. Thus, we conducted this study to develop a predictive signature based on necroptosis-related genes.

Methods: The tumor samples in The Cancer Genome Atlas (TCGA) Liver Hepatocellular Carcinoma (LIHC) cohort were subtyped using the consensus clustering algorithm. Univariate Cox regression and LASSOCox analysis were performed to construct a gene signature model from differentially expressed genes between tumor clusters. Then we integrated TNM stage and the prognostic model to build a nomogram. The gene signature and the nomogram were externally validated in the GSE14520 cohort from the gene expression omnibus (GEO) and LIRP-JP cohort from the International Cancer Genome Consortium (ICGC). Predictive performance evaluation was conducted using Kaplan-Meier plot, time-dependent receiver operating characteristic curve, principal components analysis, concordance index, and decision curve analysis. The tumor microenvironment was estimated using seven published methods. Finally, we also predicted the drug responses to immunotherapy, conventional chemotherapy and molecular-targeted therapy using two algorithms and two datasets.

Results: We identified two necroptosis-related clusters and a ten-gene signature (MTMR2, CDCA8, S100A9, ANXA10, G6PD, SLC1A5, SLC2A1, SPP1, PLOD2, and MMP1). The gene signature and the nomogram had good predictive ability in TCGA, ICGC, and GEO cohorts. The risk score was positively associated with the degree of necroptosis and immune infiltration (especially immunosuppressive cells). The high-risk group could benefit more from immunotherapy. Chemotherapy and molecular-targeted therapy should be adapted to the molecular profiles of each patient.

Conclusion: The necroptosis-related gene signature provides reliable evidence for prognosis prediction, comprehensive treatment, and new therapeutic targets for HCC patients. The nomogram can further improve predictive accuracy.

\section{Background}

Hepatocellular cancer (HCC), the fourth most common cause of cancer mortality, is projected to result in the death of more than 1 million people in 2025 worldwide [1]. Although substantial progress has been achieved in the surveillance and systemic treatment of HCC, it is still the second deadliest cancer with a dismal overall five-year survival rate of $18 \%$ [2]. Due to different etiological factors and the heterogeneous nature of HCC, prognostic prediction based on TNM strategy alone is challenging [3]. Furthermore, new therapeutic targets are urgently warranted to guide therapies and optimize medical interventions. Thus, a novel prognostic model utilizing molecular profiles of HCC is highly desirable.

Necroptosis is a newly discovered regulated cell necrosis mainly mediated by necrosome comprised of mixed lineage kinase domain-like protein (MLKL), receptor-interacting protein kinases (RIPK1) and RIPK3 [4]. Different from apoptosis in morphology, it is characterized by cell swelling and membrane perforation 
with subsequent release of intracellular damage-associated molecular patterns (DAMPs) that induce inflammation. In the context of caspase inhibition, necroptosis is initiated and functions as a backup system to combat pathogens or cancer cells that evade the apoptotic process, which is also one of the vital mechanisms for some antineoplastic drugs, such as 5-FU, etoposide, and cisplatin [5-8]. In addition to the defensive effect against malignancy, accumulating evidence suggests that necroptosis can promote tumorigenesis, cancer metastasis and immunosuppression. For example, RIPK1 is overexpressed in glioblastoma and associated with poor prognosis via attenuating p53 activation [9]. Knockout of RIPK1, RIPK3, and MLKL in the colon and esophageal cancer cells can significantly suppress tumor growth for decreased activity of NF-kappaB (NF-kB) [10]. Taken together, the role of necroptosis in cancer is highly complex and ambivalent. However, the relationship between the expressions of necroptosis-related genes and overall survival (OS) remains largely unknown in HCC patients.

In the present study, a prognostic model was established according to differentially expressed genes (DEGs) between two necroptosis-related HCC subtypes. Next, the molecular signature was integrated with the TNM staging system to build a nomogram for better predictive ability. Ultimately, we assessed the performance of risk score in forecasting tumor microenvironment (TME) and sensitivities to conventional chemotherapy, molecularly targeted therapy, and immunotherapy. Our findings provide reliable evidence for the risk stratification, comprehensive treatment, and new therapeutic targets for HCC patients.

\section{Material And Methods}

\subsection{Dataset Acquisition}

The analysis process was summarized in the flow chart (Additional file 1: Figure S1). Data of $371 \mathrm{HCC}$ samples in Liver Hepatocellular Carcinoma (LIHC) cohort were downloaded from the Cancer Genome Atlas (TCGA) repository, including fragments per kilobase of transcript per million mapped reads (FPKM) normalized values, corresponding clinical information, single nucleotide variation (SNV) and copy number variation (CNV) data (https://portal.gdc.cancer.gov/repository). Only 341 patients with follow-up time more than 30 days were subjected to prognostic model fitting. For external validation of this model, the RMA-normalized matrix of GSE14520 (including 242 tumor samples) was downloaded from Gene Expression Omnibus (GEO), and raw count data of LIRI-JP (including 231 tumor samples) from International Cancer Genome Consortium (ICGC). (https://www.ncbi.nlm.nih.gov/geo/, https://dcc.icgc.org/projects/LIRI-JP). Next, the mRNA sequencing data of TCGA-LIHC and ICGC-LIRI-JP were converted into transcripts per kilobase million (TPM) values, and then log2 $(x+1)$ transformed, which was suggested to be the most accurate quantification method with minimized statistical biases $[11,12]$. Batch effects of the above three datasets were eliminated by the "Combat" function of the "sva" package. Due to only 50 matched tumor-normal samples in TCGA-LIHC, another four datasets were included to validate the transcription levels of prognostic genes filtered by least absolute shrinkage and selection operator (LASSO)-penalized Cox regression, including GSE14520 (213 pairs), GSE25097 (243 pairs), GSE57957 (37 pairs), GSE76297 (58 pairs). 


\subsection{Investigation of the Expressions of Necroptosis-Related Genes}

Twenty-five necroptosis-related genes were extracted from the MSigDB team (GOBP_NECROPTOTIC_SIGNALING_PATHWAY) and previous reviews, which are presented in the Additional file 5: Table S1 [13-17]. SNV and CNV analyses were performed utilizing the "maftools" package and the "Rcircos" packages, respectively $[18,19]$. A network was plotted by the "igraph" package to summarize the correlation of the expression levels among necroptosis-related genes and their prognostic values in the TCGA cohort.

\subsection{Consensus Clustering}

Consensus clustering is a robust unsupervised classification technique achieved through multiple resampling and clustering. Similarity within each group was measured by Euclidean distance, and the whole process was repeated 1000 times using the "ConsensuClusterPlus" package [20]. The proper cluster number $(\mathrm{k})$ was determined according to the cumulative distribution function (CDF) plots. The flatness of the CDF curve indicates the level of consensus and the stability of clustering, which was further verified in principal components analysis (PCA) analysis.

\subsection{Identification of DEGs between two necroptosis-related clusters}

Assessment of DEG between two necroptosis-related clusters was identified by the "limma" package [21]. The false discover rate $(F D R)<0.05$ and $|\log 2 \mathrm{FC}|>1$ were criteria for statistical significance. The biological process of Gene Ontology (GO) enrichment was analyzed using the Metascape website (http://metascape.org). Based on the reference gene set available from the MSigDB (c2.cp.kegg.v7.4.symbols), we converted the DEG expression data into a pathway score matrix using the "GSVA" package [22]. The pathway activities were also compared by the "limma" package.

\subsection{Development and Validation of Necroptosis-Related Prognostic Model}

The univariate Cox analysis was carried out to select DEGs associated with overall survival. Next, LASSOpenalized Cox regression was employed to filter features to establish a risk model by the "glmnet" package $[23,24]$. The following formula was used to calculated risk score for each patient: . According to the median risk score, patients in training cohorts were classified into high- or low-risk groups. Similarly, the risk scores of external validation cohorts (ICGC and GEO) were calculated using the same formula developed from the training group and divided based on the median value of the TCGA cohort.

\subsection{Establishment and Validation of Nomogram}

We combined univariate and multivariate Cox analyses to determine the independent prognostic factors with statistical significance, which were subsequently incorporated to fit a Cox proportional hazard model [25]. For the convenience of clinical utility, a nomogram was constructed to visualize the results of the Cox model. Next, we carried out external validations in ICGC and GEO cohorts to confirm the reliability of the nomogram. The total points of each patient in the validation cohorts were calculated according to 
the Cox model derived from the TCGA cohort. Then Cox regression were implemented again using the total points as a factor, in turn, the concordance index ( $\mathrm{C}$ index) and calibration curve were yielded based on the Cox results [26]. In addition, we also plotted time-dependent receiver operating characteristic (ROC) curve and performed decision curve analysis (DCA).

\subsection{Determination of Immune Landscape}

For deciphering the immune cell infiltration, we employed single-sample gene set enrichment analysis (ssGSEA) [27] and six deconvolution algorithms to fully leverage the transcription data, including CIBERSORT [28], MCPCounter [29], xCell [30], EPIC [31], quanTIseq [32], TIMER [33]. The gene set used by SSGSEA analysis was obtained from the previous study [34], and the whole process of TME estimation was carried out using the "IOBR" package, which integrates the above seven methodologies [35].

\subsection{Prediction of drug sensitivities to chemotherapy and immunotherapy}

With an aim to provide personalized treatment recommendations, we explored the predictive value of risk score in drug sensitivities. First, molecular profiles and z-scored half-maximal inhibitory concentration (IC50) data of FDA-approved drugs were downloaded from the CellMiner database (https://discover.nci.nih.gov/cellminer) [36]. The log2(FPKM+1) data of 60 human cancer cell lines ( $\mathrm{NCl60)}$ were transformed into log2(TPM+1) values, followed by risk calculation based on the model derived from TCGA and subsequent correlation analyses. Next, we employed the "oncoPredict" package [37] to build ridge regression models to examine the differences in the efficacy of commonly used agents between high- and low-risk groups. Cancer Therapeutics Response Portal V2 (CTRP V2) database was used as the training cohort, of which the $\log 2(T P M+1)$ and half-maximal effective concentration (EC50) data were collected and shared by the "oncoPredict" team (https://osf.io/c6tfx).

As for immunotherapy, we first uploaded the normalized sequencing data of TCGA to the Tumor Immune Dysfunction and Exclusion (TIDE) website (http://tide.dfci.harvard.edu/), which is a computational prediction method based on tumor pre-treatment expression profiles [41]. Due to the lack of published data on immune checkpoint inhibitor $(\mathrm{ICl})$ in $\mathrm{HCC}$, we calculated the risk score for each patient with metastatic urothelial cancer in the IMvigor210 cohort [42]. The risk score distribution between complete/partial response (CR/PR) and stable/progressive disease (SD/PD) groups was further explored.

\subsection{Statistical Analysis}

All the data analyses and visualizations were accomplished in the R environment (Version 4.12). Wilcox statistical test was used to compare differences between two groups. Categorical data was compared using the Chi-square test. The correlation coefficient was determined by the nonparametric Spearman approach. PCA was completed by the "prcomp" function of the "stats" package. Kaplan-Meier (K-M) analysis, log-rank test, and Cox regression were performed using the "survival" and the "survminer" packages. $\mathrm{C}$ index was calculated for each Cox model and pooled using the random-effects mixed model with maximum likelihood [43]. Time-dependent ROC curve was created by the "timeROC" package to 
estimate the predictivity of each prognostic factor. The "rms" package was employed to generate the nomogram and the calibrate plot. Two-tailed $\mathrm{P}<0.05$ was considered significant.

\section{Results}

\subsection{Overview of genetic and transcriptional variation of necroptosis-related genes in HCC}

We first explored the SNV and CNV of 25 necroptosis-related genes in the TCGA-LIHC cohort. At the genetic level, 31 of 364 patients had mutations in necroptosis-related genes, of which TLR3, RIPK3 and TYRO3 showed the highest frequency (1\%), while six genes (IFNA1, IPMK, IPPK, PELI1, TRADD and TRAF2) did not display any alterations (Fig. 1A). As illustrated in Fig. 1B and 1C, genomic instability was widespread in necroptosis-related genes, including $12 \mathrm{CNV}$ gains, $12 \mathrm{CNV}$ losses, and one nonsignificant alteration. The comparison between 50 normal and $371 \mathrm{HCC}$ samples indicated that 13 genes were significantly differentially expressed, including upregulation of 12 genes and downregulation of 1 gene in tumor (Fig. 1D). The same tendency could also be observed in 50 patients with matched tumornormal samples (Fig. 1E). Additionally, we found that the gains or losses of copy number were not always positively correlated with the expression levels of pyroptosis-related genes, such as MLKL and TLR3, suggesting that CNV is not the only regulatory factor of gene expression [44]. Some mechanisms, including DNA methylation, transcription factor, m6A modification, long non-coding RNAs, and RNA binding proteins, also play vital roles in gene expression [45-48]. Fig. 1F depicts the correlations of these genes and their prognostic significances. Collectively, our results indicated that the expression of pyroptosis-related genes was significantly different between HCC and normal samples, which could be a potential contributing factor to tumorigenesis and heterogeneity.

\subsection{Identification of necroptosis-related clusters in HCC}

According to the results of k-means consensus clustering, we identified two molecular subtypes (134 patients in cluster 1 and 207 patients in cluster 2) based on necroptosis-related genes, which was also validated by PCA analysis (Fig. 2A; Additional File 2: Figure S2; Additional File 6: Table S2). Compared with cluster 2 , the overall survival (OS) of cluster 1 was significantly worse $(P<0.001)$, and most necroptosis-related genes were upregulated (Fig. 2B, C). As presented in Fig. 2E, there were significant differences between the two clusters in transcription profile, age, T stage, TNM stage, tumor grade, and gender. The top 100 of 1517 DEGs were plotted in the heatmap (Additional file 7: Table S3). Additionally, GSVA analysis revealed that the pathways enriched in the $\mathrm{C} 2$ cluster were mainly associated with metabolism, biosynthesis, and degradation. In contrast, cluster 1 showed enrichment in tumorigenesis, cell cycle, DNA replication, DNA repair, spliceosome, and immune activation, which was consistent with findings of GO enrichment (Fig. 2D, F; Additional file 9: Table S4).

\subsection{Development and validation of a ten-gene signature based on DEGs between necroptosis related clusters.}


After adjustment of batch effect, only genes shared in TCGA, ICGC and GEO cohorts were remained, so we only screened prognostic gene signature from 985 of 1517 DEGs (Additional file 3: Figure S3A). Univariate Cox regression identified 376 genes were significantly related to OS of HCC patients in TCGALIHC cohort ( $P<0.001$; Additional file 9: Table S5). Next, a 10-fold cross-validated Lasso-Cox regression was performed to determine the optimal number of genes and coefficients for the predictive model (Additional file 3: Figure S3B, C). The risk scores of TCGA, ICGC and GEO cohorts were calculated as follows: risk score $=(0.0276 *$ MTMR2 expression $)+(0.1250 *$ CDCA8 expression $)+(0.0291 *$ SLC1A5 expression $)+(0.0577 *$ G6PD expression $)+(0.0469 *$ PLOD2 expression $)+(0.0324$ * MMP1 expression $)$ $+(0.0385 *$ SLC2A1 expression $)+(-0.0136$ * ANXA10 expression $)+(0.0044 *$ S100A9 expression $)+$ (0.0050* SPP1 expression).

According to the median risk value of the TCGA cohort, patients were split into low- and high-risk groups, which were distributed in two directions in the PCA plot (Fig. 3A, E). The scatter plot indicated that risk score was negatively associated with survival time (Fig. 3B). Consistently, the K-M curves suggested that the prognosis of the high-risk group was significantly worse than that of the low-risk group $(P<0.001$; Fig. 3C). Subsequently, we performed time-dependent ROC analyses to evaluate the predictive ability of this prognostic model. The area under curves (AUCs) were $0.827 / 0.729 / 0.695$ at $1 / 3 / 5$ years in the TCGA cohort, $0.786 / 0.782 / 0.800$ at $1 / 3 / 4$ years in the ICGC cohort, and $0.645 / 0.668 / 0.668$ at $1 / 3 / 5$ years in the GEO cohort (Fig. 3D). Of note, the high-risk group was mainly composed of the $\mathrm{C} 1$ cluster and had significantly higher expression in most necroptosis-related genes, while the low-risk group predominantly consisted of the C2 cluster (Fig. 4A, B). The Sankey diagram also illustrated that the risk stratification based on the 10-gene signature was not entirely congruent with the result of the TNM system, which could provide new insight into HCC management. The Protein-protein interaction network between the ten selected genes and necroptosis-related genes is depicted in Additional file 4: Figure S3D

\subsection{External validation of the expression and genetic alteration of the ten genes}

We first investigated the transcription levels of the ten genes in the TCGA, ICGC, and GEO cohorts, finding that risk score was positively correlated with the expressions of nine genes except for AXAN10 (Fig. 5A, B, C). As depicted in the OncoPrint generated from cBioportal, 70 out of $366(19 \%)$ showed genetic alterations in the ten genes, of which amplification was the most prevalent type (Fig. 5D). Typical immunohistochemical images of nine genes were downloaded from Human Protein Atlas, except for MMP1, which was unavailable from the database (Fig. 4C). Subsequently, five datasets were used to further validate the expression levels of the ten genes between tumors and matched adjacent normal tissues (Fig. 5E-I). Interestingly, S100A9 was paradoxically overexpressed in adjacent normal tissues, while the expressions of the other nine genes were consistent with trends found between high- and lowrisk groups. S100A9 protein, also known as myeloid-related protein, typically resides in immune cells such as neutrophils, macrophages, and monocytes [49]. Although S100A9 was overexpressed in liver cancer cells, it is also intensely upregulated in myeloid cells under pathological conditions, which may account for the higher expression in adjacent normal tissue [50]. So, we further compared the tumor microenvironment of tumor and adjacent normal tissues and confirmed that the fractions of neutrophils, 
macrophages M2, and monocytes were significantly higher in adjacent normal tissues (Additional File 4: Figure S4).

\subsection{Construction and Validation of Predictive Nomogram}

Univariate and multivariate Cox analyses identified risk score and TNM stage as independent prognostic factors in TCGA, ICGC, and GEO cohorts, which were included to establish a nomogram forecasting prognosis (Fig. 6A, B, Fig. 7A). The pooled C indexes of three cohorts demonstrated that nomogram ( 0.74 , $95 \% \mathrm{Cl}: 0.69-0.79)$ had better predictive performance over risk score $(0.71,95 \% \mathrm{Cl}: 0.65-0.77)$ and TNM stage (0.66, 95\% Cl: 0.63-0.70) alone (Fig. 7B). The calibration plot illustrated nomogram achieved good consistency between the predicted and observed OS outcomes (Fig. 7C). The time-dependent ROC curves showed that the nomogram had greater AUC values, further supporting the high predictive consistency of the nomogram (Fig. 7D). According to the DCA analyses, the net benefit of the nomogram was evident in most cases (Fig. 8).

\subsection{Analyses of TME and Drug Sensitivity}

In view of the importance of risk score in prognosis prediction of HCC patients, we next investigated its potential value in reflecting TME and guiding individualized treatment. Seven published methods were used to estimate the correlation between risk score and immune infiltration in the TCGA cohort. Most cells in tumor microenvironment were positively correlated with the risk score, especially cells facilitating HCC development and immunosuppression, including type $2 \mathrm{~T}$ helper (Th2) cells, type $17 \mathrm{~T}$ helper (Th17) cells, T regulatory (Treg) cells, myeloid-derived suppressor cells (MDSCs), neutrophils, cancer-associated fibroblasts (CAFs) (Fig. 9A, C) [51,52]. Macrophages M0, B cells, dendrite cells, and T follicular helper cells exhibited the same trend. By contrast, the positive correlations between risk score and tumor-killing cells were weaker such as gamma delta $T$ cells $(T \gamma \delta), C D 8+T$ cells, natural killer $T$ (NKT) cells. In addition, we found that 37 immune checkpoint-related genes were overexpressed in the high-risk group, which further impaired the anti-tumor immunity (Fig. 9B). The risk score had positive correlations with seven genes with corresponding ICls, including PDCD1 (PD1), CD274 (PD-L1), CTLA4, LAG3, HAVCR2, TIGIT, VSIR (Fig. 9D). Subsequently, we utilized the TIDE algorithm to evaluate the response to immunotherapy. The risk score was negatively correlated with the TIDE score, and the high-risk group had a significantly lower TIDE score, suggesting that the high-risk group was more sensitive to immunotherapy (Fig.9E, F). Besides, we calculated risk scores for patients in the IMvigor210 cohort, finding that responders (CR/PR) to antiPD1/PD-L1 treatment had significantly higher risk scores (Fig. 9G).

Based on the CellMiner database, we used our prognostic model to calculate the risk scores for 60 cancer cell lines and analyzed its correlations with 218 FDA-approved drugs. A lower EC50 or IC50 value indicates a higher drug efficacy. The top 16 drugs with the most statistical differences are presented in Fig. 10. Except for rapamycin, the other 15 drugs were associated with better therapeutic effects, including oxaliplatin, parthenopid, everolimus, nitrogen mustard, actinomycin D, daunorubicin, nelarabine, cyclophosphamide, sulfatinib, lomustine, hydroxyurea, dexrazoxane, belinostat, decitabine, crizotinib. To better link the necroptosis-related gene signature with clinical practice, we used the "oncoPredict" package 
to predict commonly used drugs in the clinical treatment of HCC. The results showed that regorafenib, tivantinib, cabozantinib, cediranib, olaparib, navitoclax, mitomycin, vincristine, and paclitaxel were more sensitive in the high-risk group, while erlotinib, brivanib, dasatinib, linifanib, neratinib, fluorouracil, and methotrexate were more sensitive in the low-risk group (Fig. 11).

\section{Discussion}

Liver cancer remains a growing threat to global health. HCC accounts for approximately $90 \%$ of cases of liver cancer and is the fastest rising cause of cancer mortality [1]. Currently, the TNM staging is the most widely used system of prognosis prediction of HCC that only considers histopathological factors. Besides, given the excessive heterogeneity within HCC, individual-tailored treatment and more accurate risk stratification are critical to the improvement of therapy efficacy and overall survival. Therefore, it is highly desirable to develop a panel of molecular markers to supplement the TNM system.

Necroptosis is a pro-inflammatory form of regulated cell death and serves as an alternative defensive mechanism in the context of apoptosis inhibition caused by caspase deactivation [53]. As apoptosis escape is a well-established feature of tumors, necroptosis is consequently initiated via the formation of necrosome (RIPK1-RIPK3-MLKL complex) but may exert a double-edge sword effect on carcinogenesis [14]. For example, downregulation of MLKL implies poor prognosis in ovarian and colon cancer, while increased expression of RIP1 confers a worse prognosis in glioblastoma [54-56]. Paradoxically, one study found the pro-tumor effect of RIPK1 in HCC patients, but most human hepatoma cell lines suppress necroptosis by methylation-dependent loss of RIPK3 expression, including Huh-7, HepG2, and Hep3B. $[14,57]$. So large cohort and comprehensive analysis are warranted to investigate the role of necroptosis in HCC patients to guide treatment.

Sample classification based on the transcription profiles of predefined genes is a widely applied method. Based on the TCGA-LIHC cohort, we identified two necroptosis-related clusters and found that overexpression of these genes was related to decreased OS. To achieve better predictive ability, our prognostic model was developed from DEGs between the two subtypes rather than only from necroptosis-related genes. Consistently, the risk score was positively associated with the expression levels of most necroptosis-related genes. It has been reported that necroptosis leads to inflammation, necrosis, fibrosis, and eventually oncogenesis in patients with viral hepatitis, alcoholic liver disease, and non-alcoholic fatty liver disease [14]. Additionally, our results revealed that the degrees of infiltration of most immune cells and necroptosis were positively correlated, especially pro-tumor cells. MDSCs, Th2, and Treg cells can compromise normal immune surveillance and contribute to immune escape, while CAFs secrete growth factors and cytokines that favor tumor growth $[51,58]$. The influx of neutrophils mediated by Th17-produced interleukin-17 can drive tumor progressions via the formation of neutrophil extracellular trap (NET) $[59,60]$. Collectively, we concluded that necroptosis was more inclined to cause HCC progression instead of inhibition. 
In the present study, the novel signature was externally validated in ICGC and GEO cohorts and included two genes encoding downstream components of the necroptosis-related pathways, as RIPK1-dependent NF-KB activation will enhance the expression of S100A9 and MMP1 [61-63]. Except for MTMR2, the other nine genes were previously reported to involve in $\mathrm{HCC}$, which could be roughly classified into five categories, including cell cycle (CDCA8), chronic inflammation (S100A9), anti-oncogene (ANXA10), energy metabolism (G6PD, SLC1A5, SLC2A1), and extracellular matrix organization (SPP1, PLOD2, and MMP1). As a member of the myotubularin family of phosphoinositide lipid phosphatases, MTMR2 promotes invasion and metastasis of gastric cancer and NK/T cell lymphoma via IFNY/STAT1/IRF1 pathway and targeting JAK1, respectively $[64,65]$. CDCA8 protein is a crucial regulator of mitosis and overexpressed during hepatocarcinogenesis [66]. S100A9 is a DAMP molecule that can amplify inflammation in tumor microenvironment and lead to malignancy progression [63]. G6PD encodes glucose-6-phosphate dehydrogenase that limits the rate of the pentose phosphate pathway, which can induce EMT in HCC cells through the STAT3 pathway [67]. Increased glutamine metabolism is one of the critical metabolic modes of cancer cells [68]. Upregulation of SLC1A5 is accolated with increased uptake of glutamine and dismal prognosis in hepatic cancer [69]. Glucose transporter 1 (GLUT1) encoded by SLC2A1 is the primary regulator of glucose uptake and contributes to the metastasis and chemoresistance of liver cancer [68]. Osteopontin (OPN) was first isolated from osteoblasts and highly associated with bone mineralization [70]. In liver tissues, OPN is encoded by SPP1 and responsible for liver regeneration and correlates with the extent of liver cirrhosis and tumor growth [71]. PLOD2 protein, also known as lysyl hydroxylase, is essential for the maturation of collagen and an independent prognostic factor of HCC [72,73]. Under hypoxia environment, increased PLOD2 expression promoted migration of pancreatic cancer cells via remolding extracellular matrix [74]. Matrix metalloproteinase 1 (MMP-1) degrades the pericellular matrix, leading to enhanced invasion and metastasis [75].

There are some strengths of our studies. First, we linked necroptosis to the prognosis of HCC. Second, our prognostic model reflected the tumor immune microenvironment. Most immune cells were highly correlated with the risk score, especially pro-tumor cells. Third, our risk model could guide clinical medication. A higher risk score implied that patients could benefit more from ICI treatment. As sensitivities of drugs varied between high- and low-risk groups, chemotherapy and molecular-targeted therapy should be adapted to the sequencing result of each patient. Additionally, with the advancement of circulating tumor cell (CTC) detection and single-cell sequencing, we can also dynamically adjust the treatment regime according to the sequencing results. Fourth, the ten-gene signature and the nomogram were developed based on the TCGA cohort and further validated externally in the GEO and ICGC cohorts, which increased the reliability and stability of our results. The performance of the nomogram integrating TNM stage and 10-gene signature was superior to the two independent prognostic predictors alone.

Although we conducted comprehensive analyses and multi-database validations, several limitations inherent in this study should be noted. First, LASSO penalization would ignore some crucial genes regulating hepatocarcinogenesis. As the drug sensitivity predictions were based on bioinformatic analyses, large-scale clinical trials are warranted to confirm our findings. Moreover, the mechanism of MTMR2 in HCC remains to be elucidated for lack of relevant research. 


\section{Conclusion}

In summary, we developed a ten-gene signature based on DEGs between two necroptosis-related subtypes of $\mathrm{HCC}$ and further evaluated its predictive ability in prognosis, tumor microenvironment, and drug sensitivity. After external validation, we found that this signature was an independent prognostic factor that contributed to predicting OS and guiding clinical treatment. The Nomogram that combined histopathological and molecular features further improved the accuracy of prognostic prediction.

\section{Declarations}

\section{Acknowledgments}

We would like to thank reviewers for helpful suggestions.

\section{Funding}

This work was supported by the National Natural Science Foundation of China (No. 180530068), the special project of provincial science and technology development from National guidance (No. 2020JH6/10500055), the key research and development program of Liaoning province (2020JH2/10300130). 345 Talent Project of Shengjing Hospital of China Medical University.

\section{Abbreviations}

HCC: Hepatocellular cancer; MLKL: Mixed lineage kinase domain-like protein; RIPK: Receptor-interacting protein kinases RIPK; DAMP: damage-associated molecular pattern; NF-kB: NF-kappaB; DEG: Differentially expressed gene; TME: Tumor microenvironment; TCGA: the Cancer Genome Atlas; FPKM: Fragments per kilobase of transcript per million mapped reads; LIHC: Liver Hepatocellular Carcinoma; SNV: Single nucleotide variation; CNV: copy number variation; GEO: Gene Expression Omnibus; ICGC: International Cancer Genome Consortium; TPM: transcripts per kilobase million; LASSO: Least absolute shrinkage and selection operator; MSigDB: The Molecular Signatures Database; CDF: Cumulative distribution function; PCA: Principal components analysis; FDR: False discover rate; GO: Gene Ontology; GSVA: Gene set variation analysis; ROC: Receiver operating characteristic; DCA: decision curve analysis; C index: Concordance index; sSGSEA: Single-sample gene set enrichment analysis; IC50: maximal-inhibitory concentration; EC50: half-maximal effective concentration; FDA: Food and Drug Administration; NCI60: US National Cancer Institute 60 human tumor cell lines; CTRP: Cancer Therapeutics Response Portal; TIDE; Tumor Immune Dysfunction and Exclusion; ICl: immune checkpoint inhibitor; CR/PR: Complete/partial response; SD/PD: Stable/progressive disease; K-M: Kaplan-Meier

\section{Availability of data and materials}




\section{Ethics approval and consent to participate}

Not applicable.

\section{Competing interests}

The authors declare that they have no competing interests.

\section{Consent for publication}

Not applicable.

\section{Authors' contributions}

XDT and JLC conceived and planned this study. ZHL, JLC, HTW, ZL, and HC contributed to the interpretation of results. JLC took the lead in writing the manuscript. All authors provided vital feedback to shape the final version of the manuscript.

\section{Authors' information}

Not applicable.

\section{Author details}

${ }^{1}$ Department of General Surgery, Shengjing Hospital of China Medical University, Shenyang, Liaoning, P. R. China.

\section{References}

1. Llovet JM, Kelley RK, Villanueva A, Singal AG, Pikarsky E, Roayaie S, et al. Hepatocellular carcinoma. Nat Rev Dis Primers. 2021;7:6.

2. Nault J-C, Villanueva A. Biomarkers for Hepatobiliary Cancers. Hepatology. 2021;73 Suppl 1:115-27.

3. Yang JD, Hainaut P, Gores GJ, Amadou A, Plymoth A, Roberts LR. A global view of hepatocellular carcinoma: trends, risk, prevention and management. Nat Rev Gastroenterol Hepatol. 2019;16:589604. 
4. Scarpitta A, Hacker UT, Büning H, Boyer O, Adriouch S. Pyroptotic and Necroptotic Cell Death in the Tumor Microenvironment and Their Potential to Stimulate Anti-Tumor Immune Responses. Front Oncol. 2021;11:731598.

5. Schwabe RF, Luedde T. Apoptosis and necroptosis in the liver: a matter of life and death. Nat Rev Gastroenterol Hepatol. 2018;15:738-52.

6. Xu Y, Ma H-B, Fang Y-L, Zhang Z-R, Shao J, Hong M, et al. Cisplatin-induced necroptosis in TNFa dependent and independent pathways. Cell Signal. 2017;31:112-23.

7. Brown MF, Leibowitz BJ, Chen D, He K, Zou F, Sobol RW, et al. Loss of caspase-3 sensitizes colon cancer cells to genotoxic stress via RIP1-dependent necrosis. Cell Death Dis. 2015;6:e1729.

8. Tenev T, Bianchi K, Darding M, Broemer M, Langlais C, Wallberg F, et al. The Ripoptosome, a signaling platform that assembles in response to genotoxic stress and loss of IAPs. Mol Cell. 2011;43:432-48.

9. Park S, Hatanpaa KJ, Xie Y, Mickey BE, Madden CJ, Raisanen JM, et al. The receptor interacting protein 1 inhibits p53 induction through NF-kappaB activation and confers a worse prognosis in glioblastoma. Cancer Res. 2009;69:2809-16.

10. Liu X, Zhou M, Mei L, Ruan J, Hu Q, Peng J, et al. Key roles of necroptotic factors in promoting tumor growth. Oncotarget. 2016;7:22219-33.

11. Wagner GP, Kin K, Lynch VJ. Measurement of mRNA abundance using RNA-seq data: RPKM measure is inconsistent among samples. Theory Biosci. 2012;131:281-5.

12. Jin H, Wan Y-W, Liu Z. Comprehensive evaluation of RNA-seq quantification methods for linearity. BMC Bioinformatics. 2017;18:117.

13. Post SM, Andreeff M, DiNardo C, Khoury JD, Ruvolo PP. TAM kinases as regulators of cell death. Biochim Biophys Acta Mol Cell Res. 2021;1868:118992.

14. Li X, Dong G, Xiong H, Diao H. A narrative review of the role of necroptosis in liver disease: a doubleedged sword. Ann Transl Med. 2021;9:422.

15. Lou J, Zhou Y, Feng Z, Ma M, Yao Y, Wang Y, et al. Caspase-Independent Regulated Necrosis Pathways as Potential Targets in Cancer Management. Front Oncol. 2020;10:616952.

16. de Miguel D, Ramirez-Labrada A, Uranga I, Hidalgo S, Santiago L, Galvez EM, et al. Inflammatory cell death induced by cytotoxic lymphocytes: a dangerous but necessary liaison. FEBS J. 2021;

17. Karlowitz R, Wijk SJL. Surviving death: emerging concepts of RIPK3 and MLKL ubiquitination in the regulation of necroptosis. FEBS J. 2021;febs.16255.

18. Mayakonda A, Lin D-C, Assenov Y, Plass C, Koeffler HP. Maftools: efficient and comprehensive analysis of somatic variants in cancer. Genome Res. 2018;28:1747-56.

19. Zhang H, Meltzer P, Davis S. RCircos: an R package for Circos 2D track plots. BMC Bioinformatics. 2013;14:244.

20. Wilkerson MD, Hayes DN. ConsensusClusterPlus: a class discovery tool with confidence assessments and item tracking. Bioinformatics. 2010;26:1572-3. 
21. Ritchie ME, Phipson B, Wu D, Hu Y, Law CW, Shi W, et al. limma powers differential expression analyses for RNA-sequencing and microarray studies. Nucleic Acids Res. 2015;43:e47.

22. Hänzelmann S, Castelo R, Guinney J. GSVA: gene set variation analysis for microarray and RNA-seq data. BMC Bioinformatics. 2013;14:7.

23. Tibshirani R. The lasso method for variable selection in the Cox model. Stat Med. 1997;16:385-95.

24. N S, J F, T H, R T. Regularization Paths for Cox's Proportional Hazards Model via Coordinate Descent. Journal of statistical software [Internet]. J Stat Softw; 2011 [cited 2021 Nov 13];39. Available from: https://pubmed.ncbi.nlm.nih.gov/27065756/

25. Iasonos A, Schrag D, Raj GV, Panageas KS. How to build and interpret a nomogram for cancer prognosis. J Clin Oncol. 2008;26:1364-70.

26. Prognostic Nomogram for Intrahepatic Cholangiocarcinoma After PartialWang et al_2013_.pdf [Internet]. [cited 2021 Nov 24]. Available from: https://moscow.scihub.se/2465/eb6635088f773b171a7c6760dae8901e/wang2013.pdf\#navpanes=0\&view=FitH

27. Hänzelmann S, Castelo R, Guinney J. GSVA: gene set variation analysis for microarray and RNA-seq data. BMC Bioinformatics. 2013;14:7.

28. Newman AM, Liu CL, Green MR, Gentles AJ, Feng W, Xu Y, et al. Robust enumeration of cell subsets from tissue expression profiles. Nat Methods. 2015;12:453-7.

29. Becht E, Giraldo NA, Lacroix L, Buttard B, Elarouci N, Petitprez F, et al. Estimating the population abundance of tissue-infiltrating immune and stromal cell populations using gene expression. Genome Biol. 2016;17:218.

30. Aran D, Hu Z, Butte AJ. xCell: digitally portraying the tissue cellular heterogeneity landscape. Genome Biol. 2017;18:220.

31. Racle J, de Jonge K, Baumgaertner P, Speiser DE, Gfeller D. Simultaneous enumeration of cancer and immune cell types from bulk tumor gene expression data. Elife. 2017;6:e26476.

32. Finotello F, Mayer C, Plattner C, Laschober G, Rieder D, Hackl H, et al. Molecular and pharmacological modulators of the tumor immune contexture revealed by deconvolution of RNA-seq data. Genome Med. 2019;11:34.

33. Li B, Liu JS, Liu XS. Revisit linear regression-based deconvolution methods for tumor gene expression data. Genome Biol. 2017;18:127.

34. Charoentong P, Finotello F, Angelova M, Mayer C, Efremova M, Rieder D, et al. Pan-cancer Immunogenomic Analyses Reveal Genotype-Immunophenotype Relationships and Predictors of Response to Checkpoint Blockade. Cell Reports. 2017;18:248-62.

35. Zeng D, Ye Z, Shen R, Yu G, Wu J, Xiong Y, et al. IOBR: Multi-Omics Immuno-Oncology Biological Research to Decode Tumor Microenvironment and Signatures. Front Immunol. 2021;12:687975.

36. Shankavaram UT, Varma S, Kane D, Sunshine M, Chary KK, Reinhold WC, et al. CellMiner: a relational database and query tool for the NCl-60 cancer cell lines. BMC Genomics. 2009;10:277. 
37. Maeser D, Gruener RF, Huang RS. oncoPredict: an R package for predicting in vivo or cancer patient drug response and biomarkers from cell line screening data. Brief Bioinform. 2021;22:bbab260.

38. Basu A, Bodycombe NE, Cheah JH, Price EV, Liu K, Schaefer GI, et al. An interactive resource to identify cancer genetic and lineage dependencies targeted by small molecules. Cell. 2013;154:115161.

39. Mg R, B S-L, Jh C, Dj A, Ev P, S G, et al. Correlating chemical sensitivity and basal gene expression reveals mechanism of action. Nature chemical biology [Internet]. Nat Chem Biol; 2016 [cited 2021 Dec 10];12. Available from: https://pubmed.ncbi.nlm.nih.gov/26656090/

40. B S-L, Mg R, Jh C, M C, Ev P, Me C, et al. Harnessing Connectivity in a Large-Scale Small-Molecule Sensitivity Dataset. Cancer discovery [Internet]. Cancer Discov; 2015 [cited 2021 Dec 10];5. Available from: https://pubmed.ncbi.nIm.nih.gov/26482930/

41. Jiang P, Gu S, Pan D, Fu J, Sahu A, Hu X, et al. Signatures of T cell dysfunction and exclusion predict cancer immunotherapy response. Nat Med. 2018;24:1550-8.

42. Mariathasan S, Turley SJ, Nickles D, Castiglioni A, Yuen K, Wang Y, et al. TGF $\beta$ attenuates tumour response to PD-L1 blockade by contributing to exclusion of T cells. Nature. 2018;554:544-8.

43. Pinheiro JC, Bates DM. Approximations to the Log-Likelihood Function in the Nonlinear MixedEffects Model. Journal of Computational and Graphical Statistics. Taylor \& Francis; 1995;4:12-35.

44. Gentles AJ, Newman AM, Liu CL, Bratman SV, Feng W, Kim D, et al. The prognostic landscape of genes and infiltrating immune cells across human cancers. Nat Med. 2015;21:938-45.

45. Romero IG, Ruvinsky I, Gilad Y. Comparative studies of gene expression and the evolution of gene regulation. Nat Rev Genet. 2012;13:505-16.

46. Coker $\mathrm{H}$, Wei G, Brockdorff N. m6A modification of non-coding RNA and the control of mammalian gene expression. Biochim Biophys Acta Gene Regul Mech. 2019;1862:310-8.

47. Gil N, Ulitsky I. Regulation of gene expression by cis-acting long non-coding RNAs. Nat Rev Genet. 2020;21:102-17.

48. Corbett AH. Post-transcriptional regulation of gene expression and human disease. Curr Opin Cell Biol. 2018;52:96-104.

49. Shabani F, Farasat A, Mahdavi M, Gheibi N. Calprotectin (S100A8/S100A9): a key protein between inflammation and cancer. Inflamm Res. 2018;67:801-12.

50. Gebhardt C, Németh J, Angel P, Hess J. S100A8 and S100A9 in inflammation and cancer. Biochem Pharmacol. 2006;72:1622-31.

51. Lu C, Rong D, Zhang B, Zheng W, Wang X, Chen Z, et al. Current perspectives on the immunosuppressive tumor microenvironment in hepatocellular carcinoma: challenges and opportunities. Mol Cancer. 2019;18:130.

52. Lan Y-T, Fan X-P, Fan Y-C, Zhao J, Wang K. Change in the Treg/Th17 cell imbalance in hepatocellular carcinoma patients and its clinical value. Medicine (Baltimore). 2017;96:e7704. 
53. Zhu F, Zhang W, Yang T, He S-D. Complex roles of necroptosis in cancer. J Zhejiang Univ Sci B. 2019;20:399-413.

54. He L, Peng K, Liu Y, Xiong J, Zhu F-F. Low expression of mixed lineage kinase domain-like protein is associated with poor prognosis in ovarian cancer patients. Onco Targets Ther. 2013;6:1539-43.

55. Li X, Guo J, Ding A-P, Qi W-W, Zhang P-H, Lv J, et al. Association of Mixed Lineage Kinase DomainLike Protein Expression With Prognosis in Patients With Colon Cancer. Technol Cancer Res Treat. 2017;16:428-34.

56. Park S, Hatanpaa KJ, Xie Y, Mickey BE, Madden CJ, Raisanen JM, et al. The receptor interacting protein 1 inhibits $p 53$ induction through NF-kappaB activation and confers a worse prognosis in glioblastoma. Cancer Res. 2009;69:2809-16.

57. Wang $C$, Yao B, Xu M, Zheng X. RIP1 upregulation promoted tumor progression by activating $\mathrm{AKT} / \mathrm{BCl}-2 / \mathrm{BAX}$ signaling and predicted poor postsurgical prognosis in HCC. Tumour Biol. 2016;37:15305-13.

58. Kogame $M$, Nagai $H$, Shinohara $M$, Igarashi $Y$, Sumino $Y$, Ishii K. Th2 Dominance Might Induce Carcinogenesis in Patients with HCV-related Liver Cirrhosis. Anticancer Res. 2016;36:4529-36.

59. Nakae S, Suto H, Berry GJ, Galli SJ. Mast cell-derived TNF can promote Th17 cell-dependent neutrophil recruitment in ovalbumin-challenged OTII mice. Blood. 2007;109:3640-8.

60. Hilscher MB, Shah VH. Neutrophil Extracellular Traps and Liver Disease. Semin Liver Dis. 2020;40:171-9.

61. Meylan E, Tschopp J. The RIP kinases: crucial integrators of cellular stress. Trends Biochem Sci. 2005;30:151-9.

62. Nguyen CH, Senfter D, Basilio J, Holzner S, Stadler S, Krieger S, et al. NF-kB contributes to MMP1 expression in breast cancer spheroids causing paracrine PAR1 activation and disintegrations in the lymph endothelial barrier in vitro. Oncotarget. 2015;6:39262-75.

63. Duan L, Wu R, Zhang X, Wang D, You Y, Zhang Y, et al. HBx-induced S100A9 in NF-kB dependent manner promotes growth and metastasis of hepatocellular carcinoma cells. Cell Death Dis. 2018;9:629.

64. Jiang L, Liu J-Y, Shi Y, Tang B, He T, Liu J-J, et al. MTMR2 promotes invasion and metastasis of gastric cancer via inactivating IFNY/STAT1 signaling. J Exp Clin Cancer Res. 2019;38:206.

65. Wang J, He A-L, Zhang W-G, Cao X-M, Chen Y-X, Liu J, et al. MTMR2 promotes the progression of NK/T cell lymphoma by targeting JAK1. Eur Rev Med Pharmacol Sci. 2020;24:8057-66.

66. Li L, Li D, Tian F, Cen J, Chen X, Ji Y, et al. Hepatic Loss of Borealin Impairs Postnatal Liver Development, Regeneration, and Hepatocarcinogenesis. J Biol Chem. 2016;291:21137-47.

67. Lu M, Lu L, Dong Q, Yu G, Chen J, Qin L, et al. Elevated G6PD expression contributes to migration and invasion of hepatocellular carcinoma cells by inducing epithelial-mesenchymal transition. Acta Biochim Biophys Sin (Shanghai). 2018;50:370-80. 
68. Amann T, Hellerbrand C. GLUT1 as a therapeutic target in hepatocellular carcinoma. Expert Opin Ther Targets. 2009;13:1411-27.

69. Zhang P, Wang Q, Lin Z, Yang P, Dou K, Zhang R. Berberine Inhibits Growth of Liver Cancer Cells by Suppressing Glutamine Uptake. Onco Targets Ther. 2019;12:11751-63.

70. Butler WT. The nature and significance of osteopontin. Connect Tissue Res. 1989;23:123-36.

71. Song Z, Chen W, Athavale D, Ge X, Desert R, Das S, et al. Osteopontin Takes Center Stage in Chronic Liver Disease. Hepatology. 2021;73:1594-608.

72. Noda T, Yamamoto $H$, Takemasa I, Yamada D, Uemura M, Wada H, et al. PLOD2 induced under hypoxia is a novel prognostic factor for hepatocellular carcinoma after curative resection. Liver Int. 2012;32:110-8.

73. van der Slot AJ, Zuurmond A-M, Bardoel AFJ, Wijmenga C, Pruijs HEH, Sillence DO, et al. Identification of PLOD2 as telopeptide lysyl hydroxylase, an important enzyme in fibrosis. J Biol Chem. 2003;278:40967-72.

74. Sada M, Ohuchida K, Horioka K, Okumura T, Moriyama T, Miyasaka Y, et al. Hypoxic stellate cells of pancreatic cancer stroma regulate extracellular matrix fiber organization and cancer cell motility. Cancer Lett. 2016;372:210-8.

75. Liang Y, Clay NE, Sullivan KM, Leong J, Ozcelikkale A, Rich MH, et al. Enzyme-Induced Matrix Softening Regulates Hepatocarcinoma Cancer Cell Phenotypes. Macromol Biosci. 2017;17.

\section{Figures}



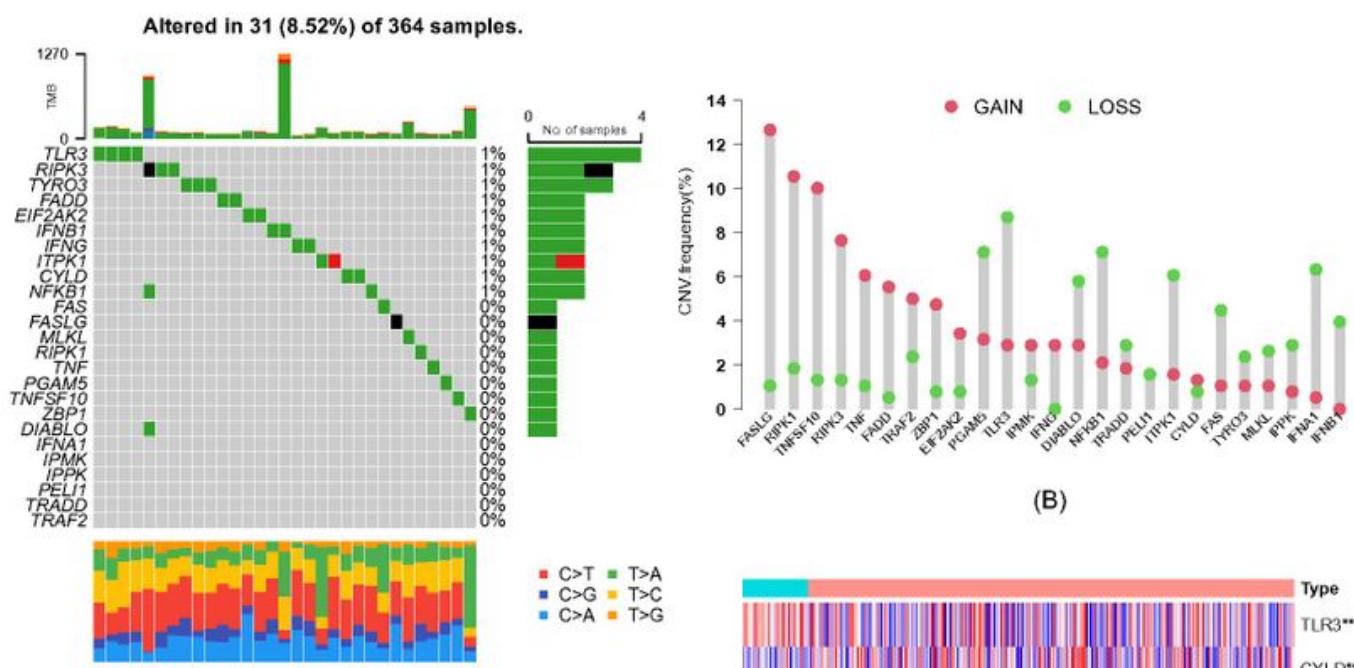

$\| C>T=T>A$
$=C>G=T>C$
$=C>A=T>G$

(B)

(A)
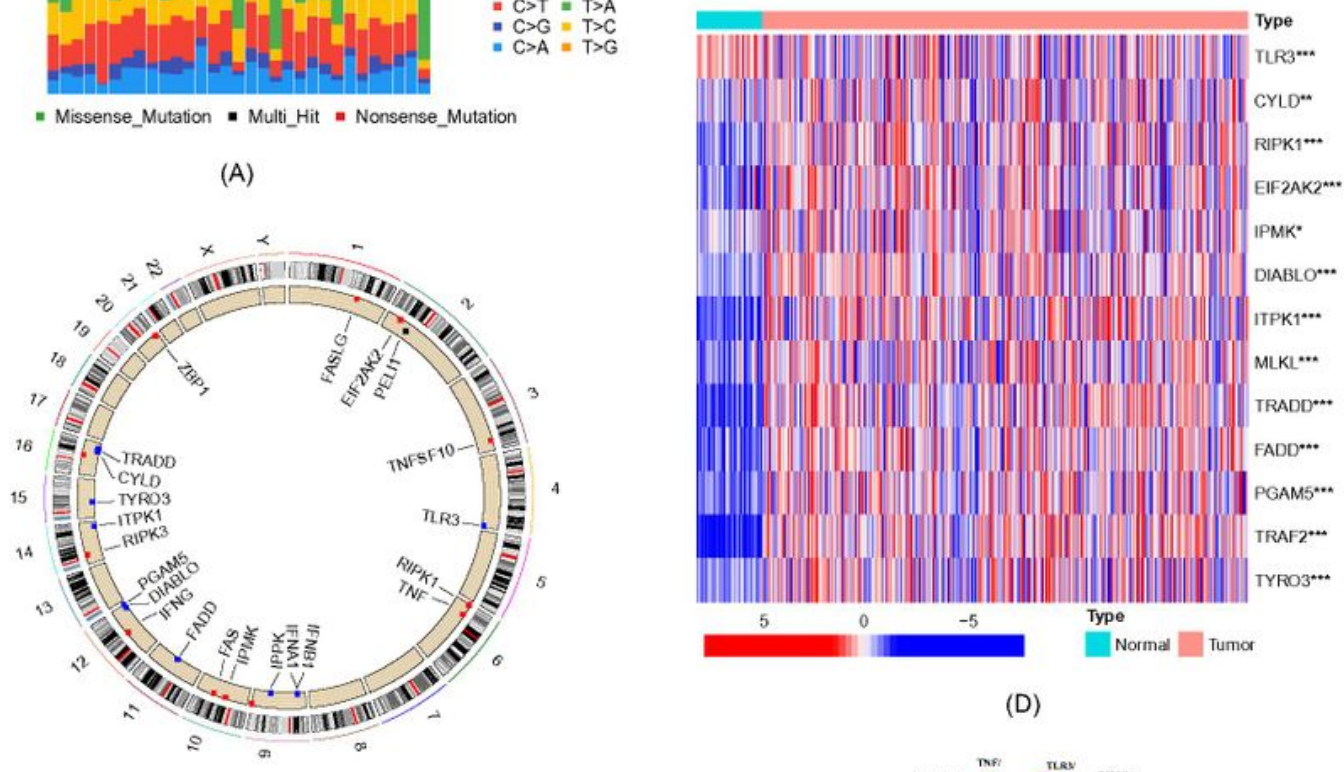

(D)

(C)

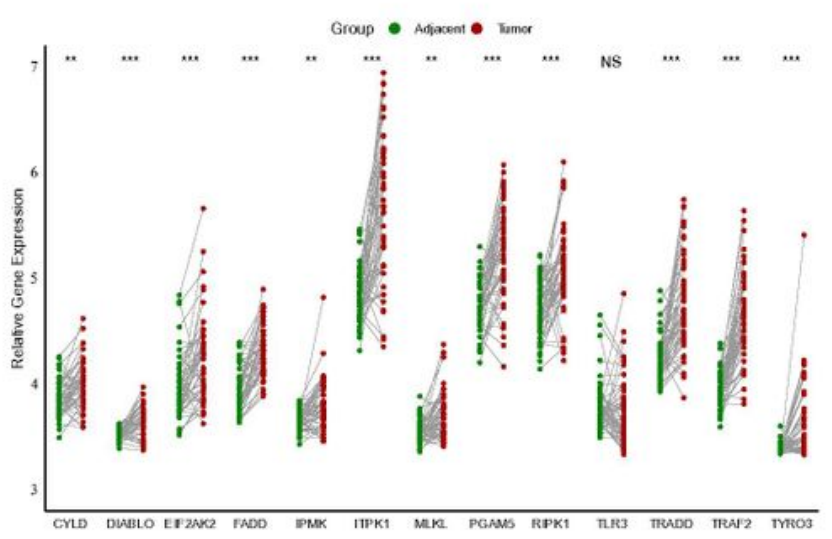

(E)

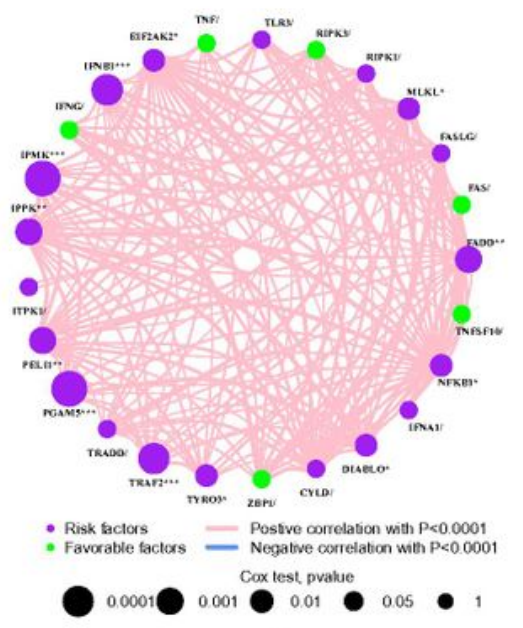

(F)

\section{Figure 1}

Genetic and transcriptional variation of necroptosis-related genes in TCGA-LIHC cohort.

(A) Oncoplot depicting the mutations of necroptosis-related genes. (B) Copy number variation (CNV) frequency of pyroptosis-related genes. (C) The location of necroptosis-related genes with CNV alteration on chromosomes. (D) The heatmap of differentially expressed necroptosis-related genes between tumor 
and normal samples (Wilcox test, $* \mathrm{P}<0.05 ; * * \mathrm{P}<0.01 ; * * * \mathrm{P}<0.001 ; * * * * \mathrm{P}<0.0001$ ). (E) The mRNA levels of 13 necroptosis-related genes in 50 matched tumor-normal samples (Wilcox test, $* \mathrm{P}<$ $0.05 ; * * \mathrm{P}<0.01 ; * * * \mathrm{P}<0.001 ; * * * * \mathrm{P}<0.0001)$. (F) A network plot illustrating the correlations of necroptosis-related genes and their prognostic values.

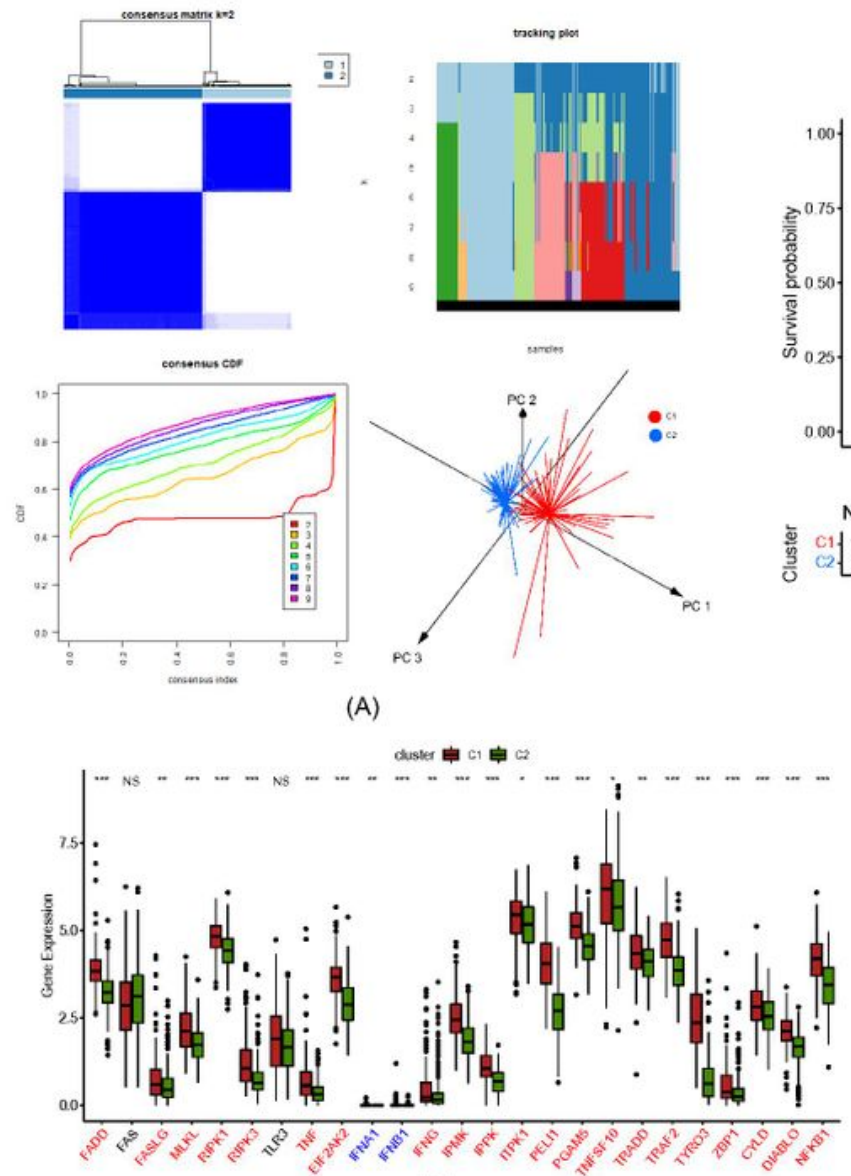

(C)

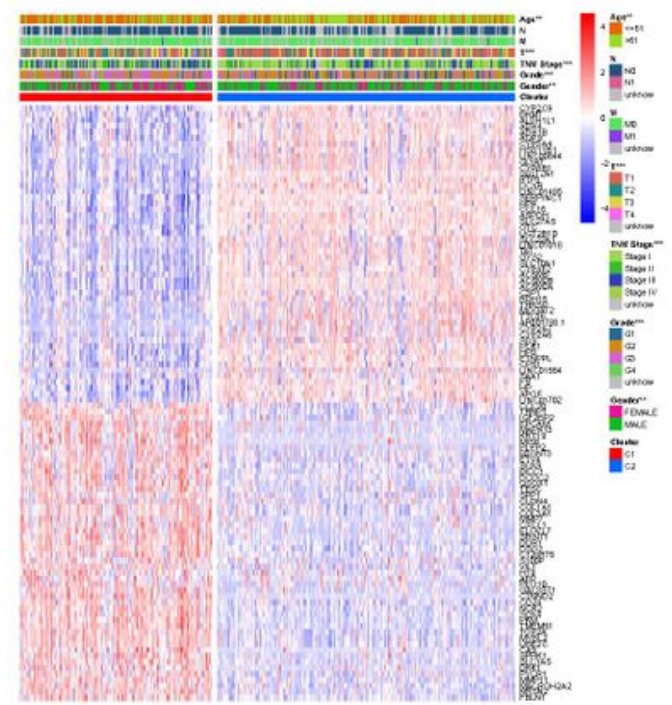

(E)
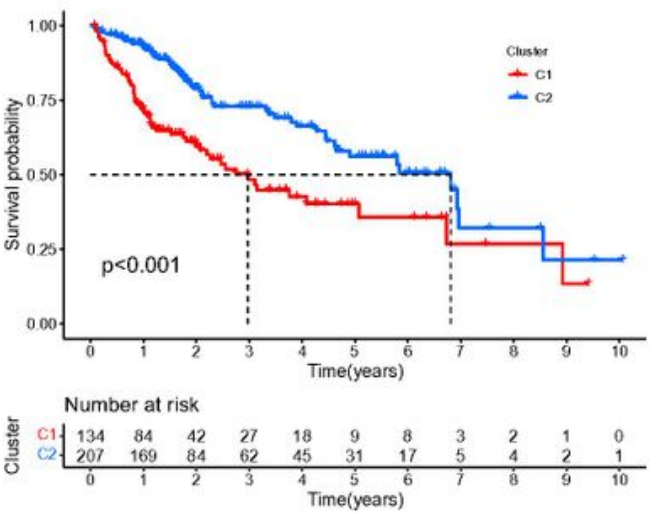

(B)

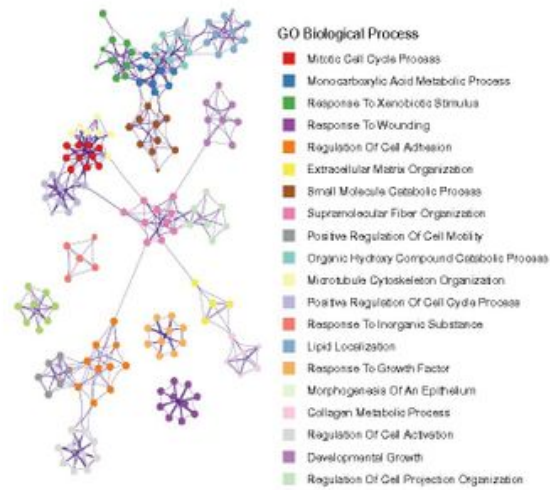

(D)

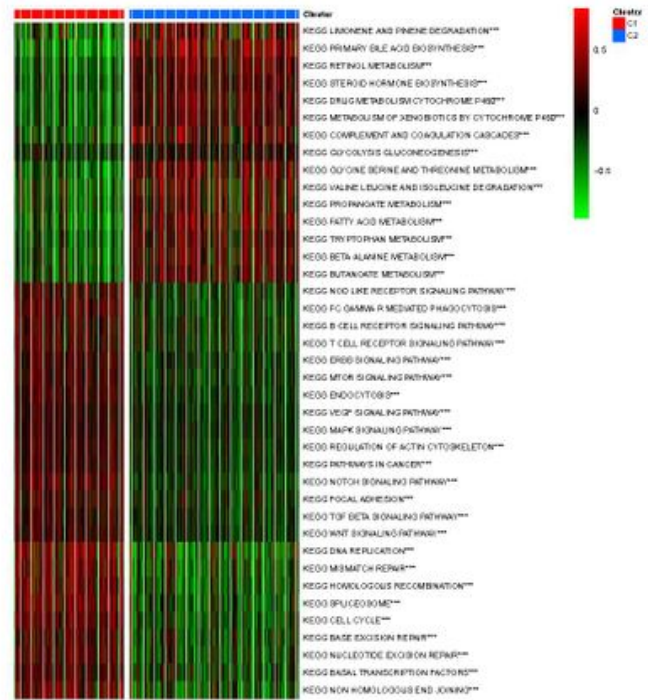

(F)

Figure 2 
Identification and characteristics of necroptosis-related subgroups in TCGA-LIHC cohort.

(A) Consensus matrix heatmap when $\mathrm{k}=2$; Tracking plot depicting the assignment of items (in columns) at each $\mathrm{k}$ (in rows); Cumulative distribution function (CDF) curve illustrating the stability at each $\mathrm{k}$; principal component analysis (PCA) plot showing the difference between two clusters. (B) Kaplan-Meier curve assessed the difference in overall survival between the two clusters. (C) The expression levels of necroptosis-related genes between two clusters (Wilcox test, $* \mathrm{P}<0.05 ; * * \mathrm{P}<0.01 ; * * * \mathrm{P}<0.001$; $* * * * \mathrm{P}<0.0001$; NS, not statistically significant). (D) The biological process enrichment using Metascape website. (E) Differences in clinicopathologic features and expression levels of necroptosisrelated between the two clusters (Bayes moderation, $* \mathrm{P}<0.05 ; * * \mathrm{P}<0.01 ; * * * \mathrm{P}<0.001 ; * * * * \mathrm{P}<$ 0.0001 ). The top 100 of $1517 \mathrm{DEGs}$ were plotted in the heatmap. (F) Differences in active pathways between two clusters (Bayes moderation, $* \mathrm{P}<0.05 ; * * \mathrm{P}<0.01 ; * * * \mathrm{P}<0.001 ; * * * * \mathrm{P}<0.0001$ ). 

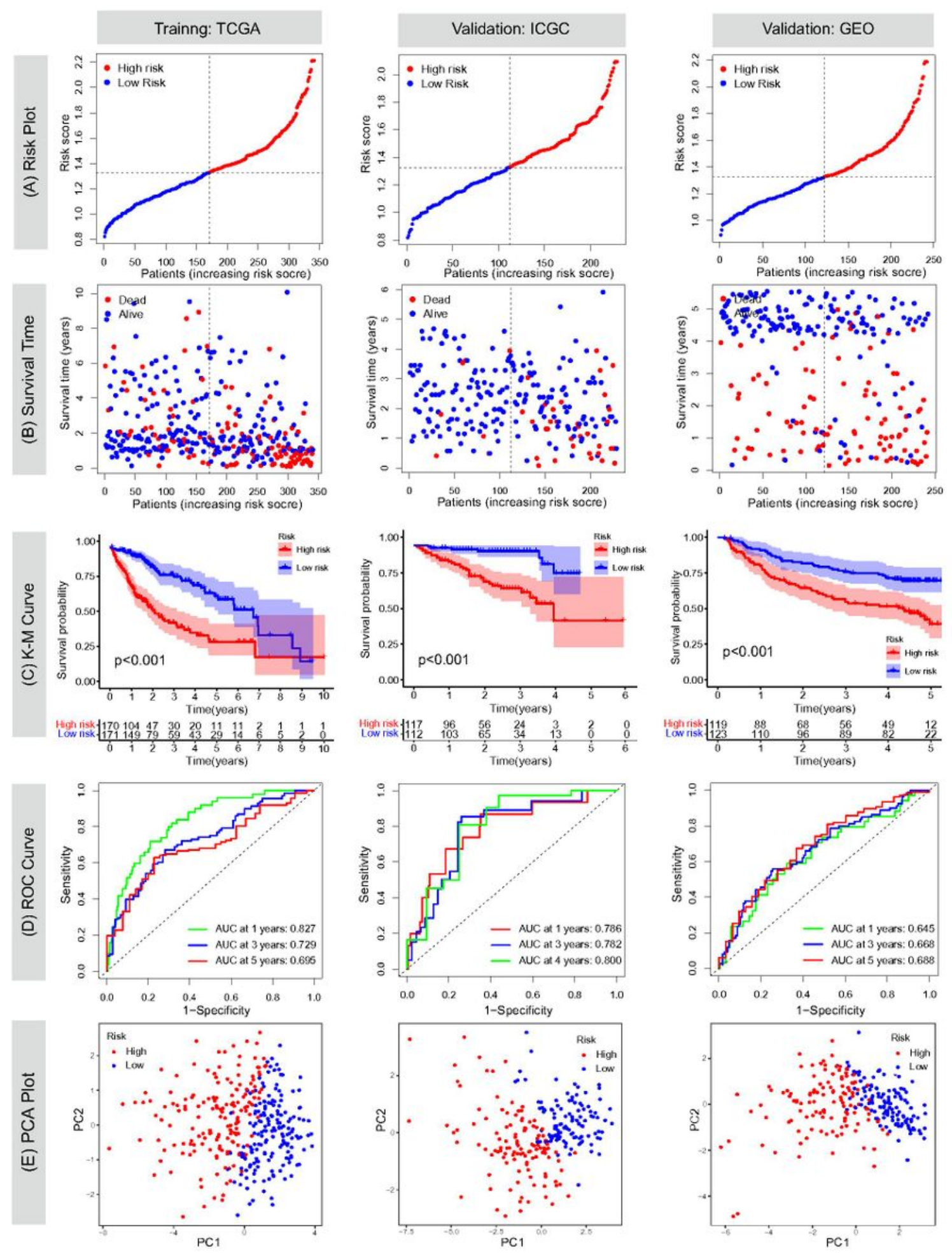

\section{Figure 3}

Prognostic values of the ten-gene signature in TCGA-LIHC, ICGC-LIRI-JP, and GSE14520 cohorts.

(A) median values of risk scores and their distributions. (B) The correlations of risk scores and overall survivals. (C) Kaplan-Meier curves assessed the differences in overall survival between high- and low- 
risk groups. (D) Time-dependent receiver operating characteristic (ROC) curves and area under curve (AUC) analyses. (E) Principal component analysis (PCA) plots.

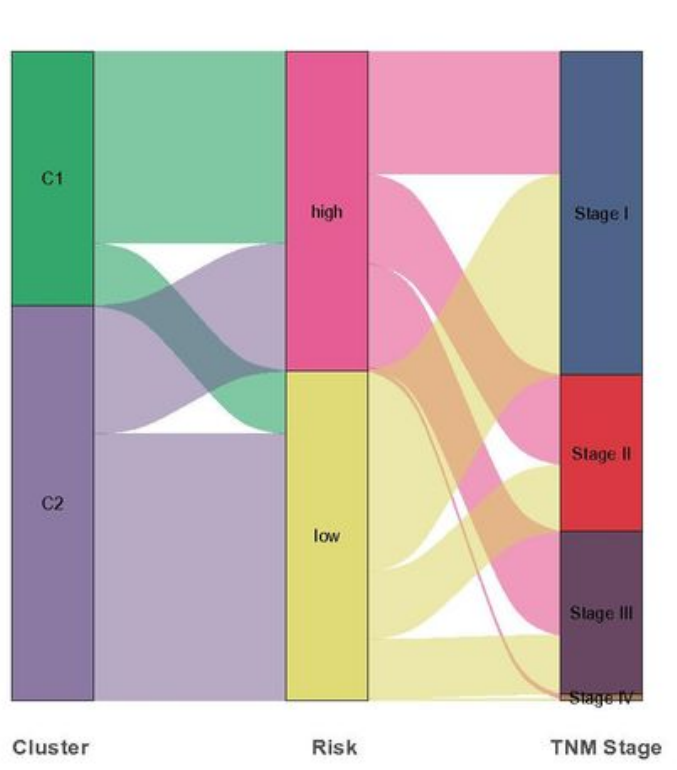

(A)

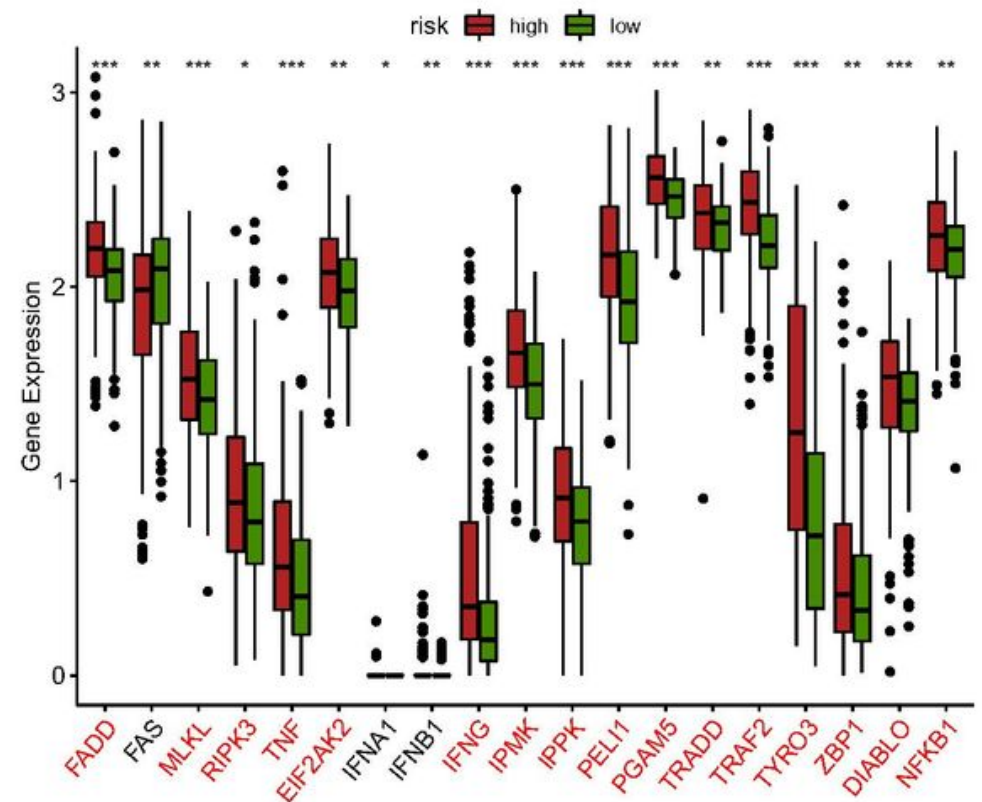

(B)

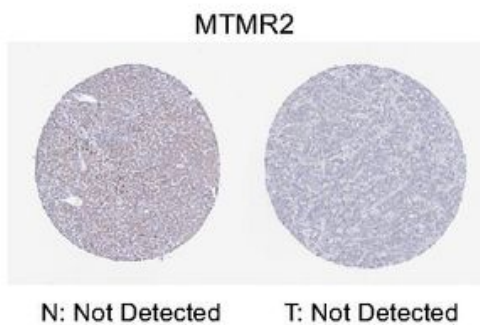

G6PD

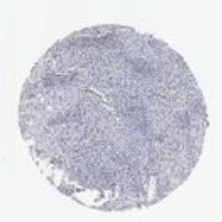

N: Not Detected

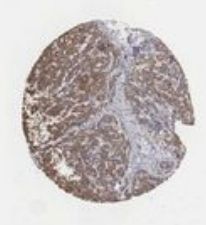

T: High

ANXA10

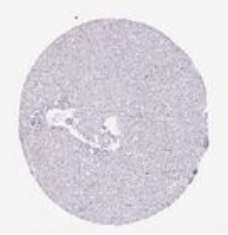

$\mathrm{N}$ : Not Detected

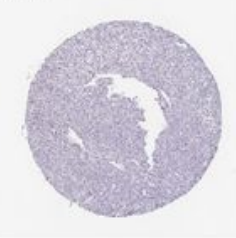

T: Not Detected

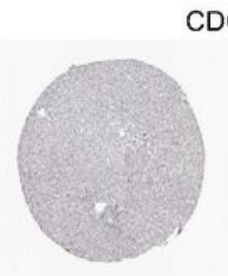

N: Not Detected

PLOD2

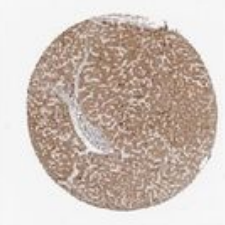

$\mathrm{N}$ : Medium

S100A9

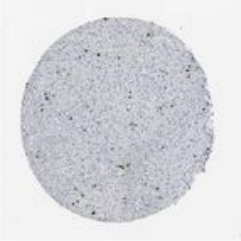

$\mathrm{N}$ : Not Detected
CDCA8

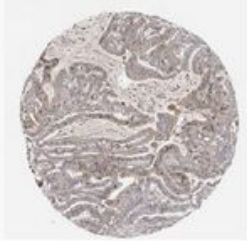

T: High

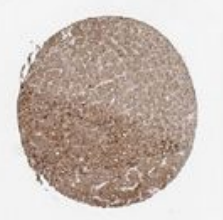

T: Medium

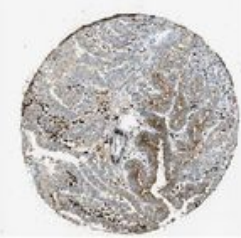

T: High

SLC1A5

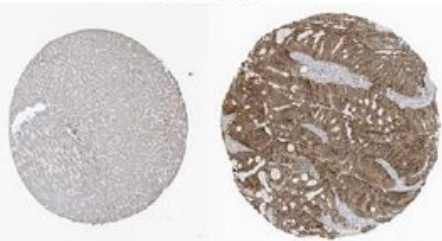

$\mathrm{N}$ : Not Detected

T: High

SLC2A1

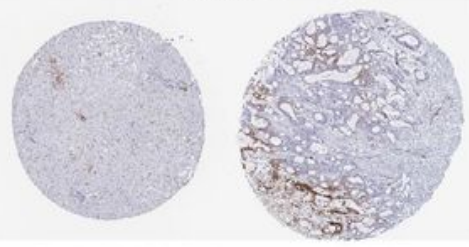

N: Not Detected

T: High

SPP1

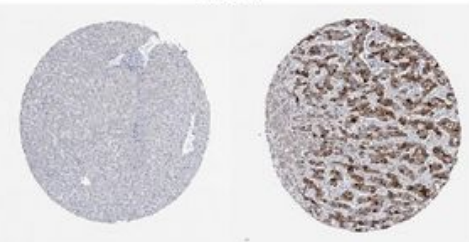

N: Not Detected

T: High

(C)

Figure 4

The association between gene signature and necroptosis 
(A) Sankey diagram depicting the association between tumor cluster, risk score, and TNM stage in TCGALIHC cohort. (B) The expression levels of necroptosis-related genes between high- and low-risk groups in TCGA-LIHC cohort (Wilcox test, $* \mathrm{P}<0.05 ; * * \mathrm{P}<0.01 ; * * * \mathrm{P}<0.001 ; * * * * \mathrm{P}<0.0001$ ). Only genes with statistical significance were displayed in this plot. (C) Immunohistochemical images of the nine genes in the risk model obtained from the Human Protein Atlas (https://www.proteinatlas.org/).

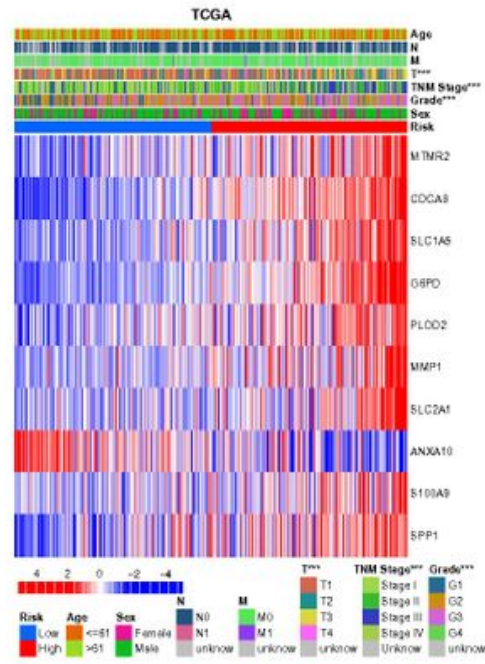

(A)

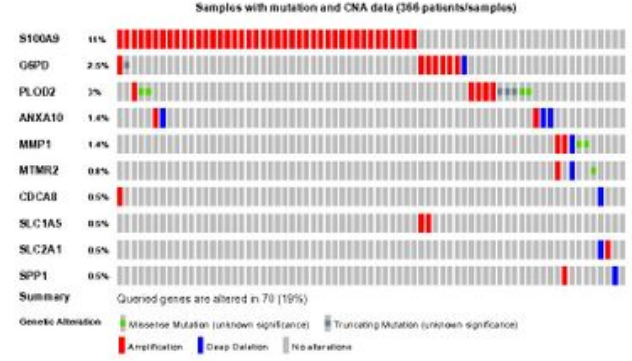

(D)
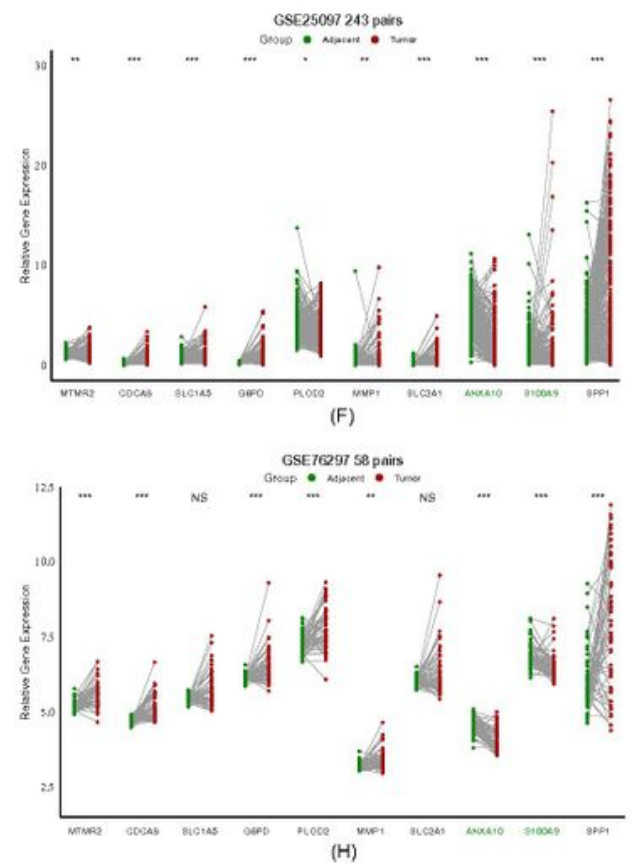

ICGC

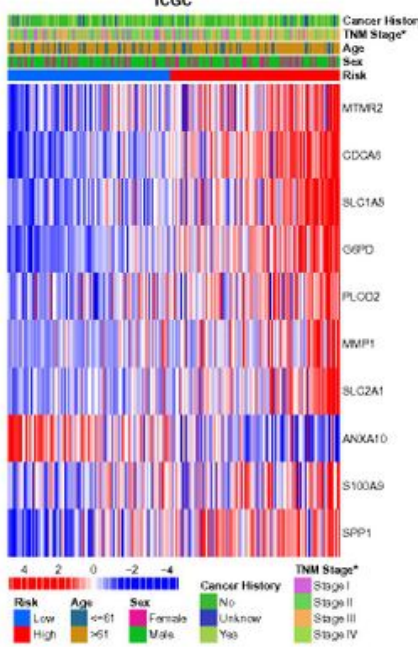

(B)

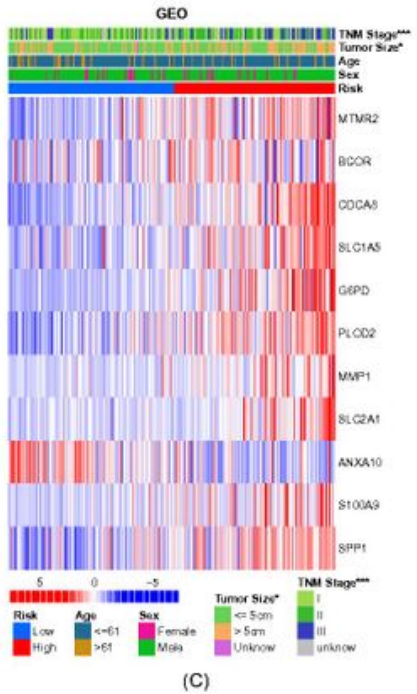

TCGA-LIHC SO pairs
Growp
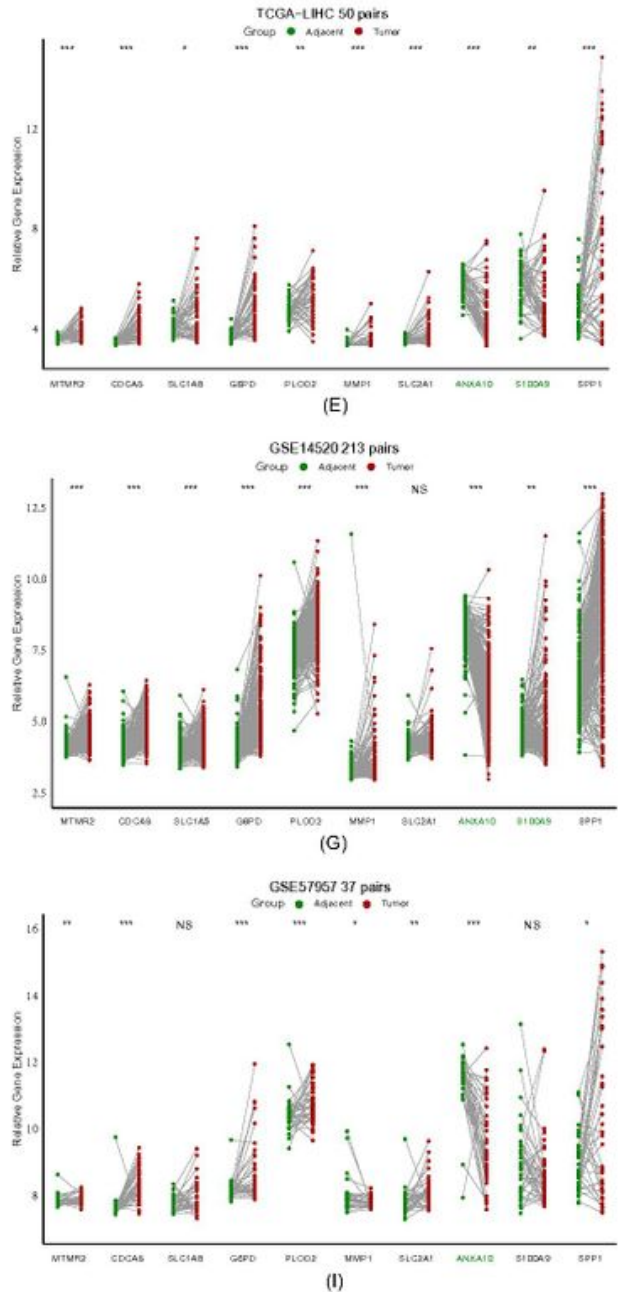

Figure 5 
The expression and genetic alteration of the ten genes included in the risk model.

(A-C) The transcription levels of the ten genes and clinical characteristics between high- and low-risk groups in the TCGA-LIHC, ICGC-LIRI-JP, and GSE1450 cohorts. (D) Genetic alterations of the ten genes in TCGA-LIHC. The OncoPrint was downloaded from the cBioportal (https://www.cbioportal.org/). (E-I) Validations of expression levels of the ten genes between tumors and matched adjacent normal tissues in TCGA-LIHC, GSE14520, GSE25097, GSE57957, GSE76297cohorts.

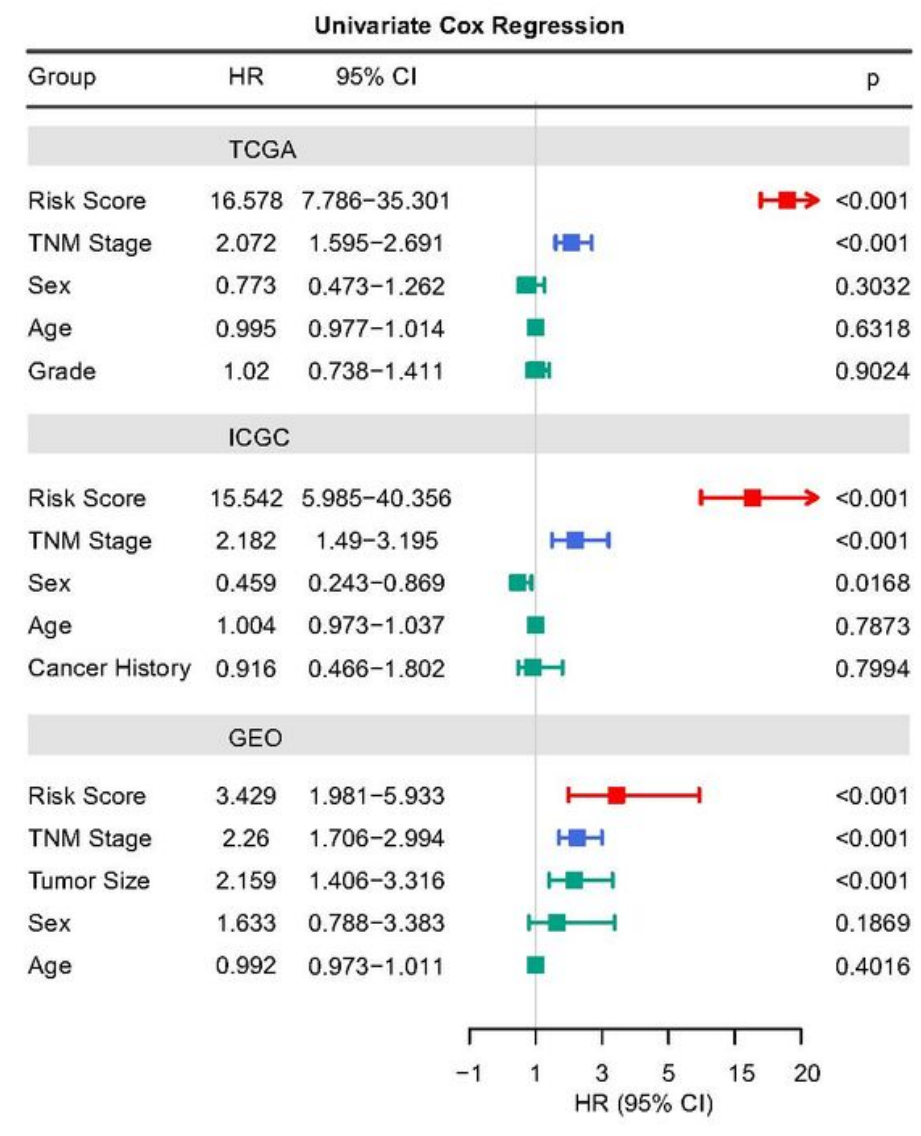

(A)

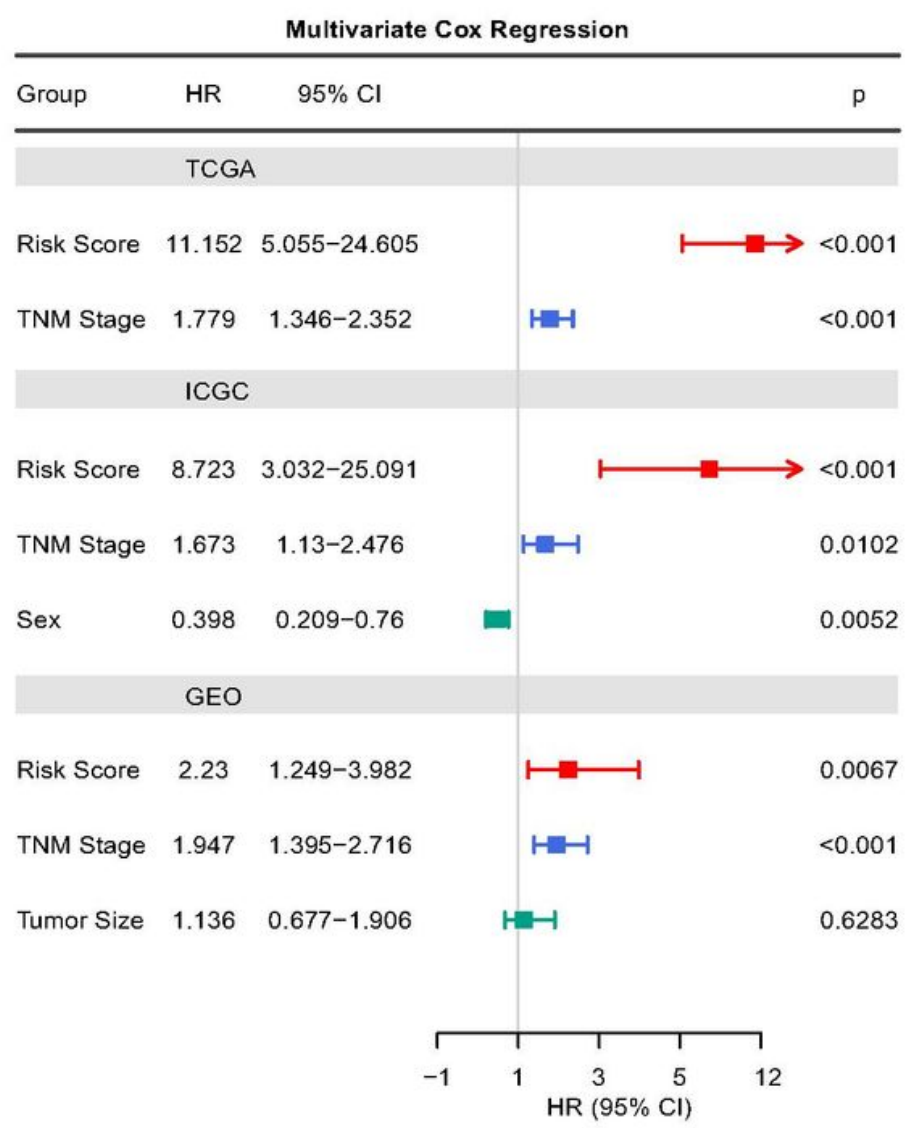

(B)

\section{Figure 6}

Identification of independent prognostic factors in TCGA-LIHC, ICGC-LIRI-JP, and GSE1450 cohorts.

(A) Results of the univariate Cox regression analyses. (B) Results of the multivariate Cox regression analyses. 


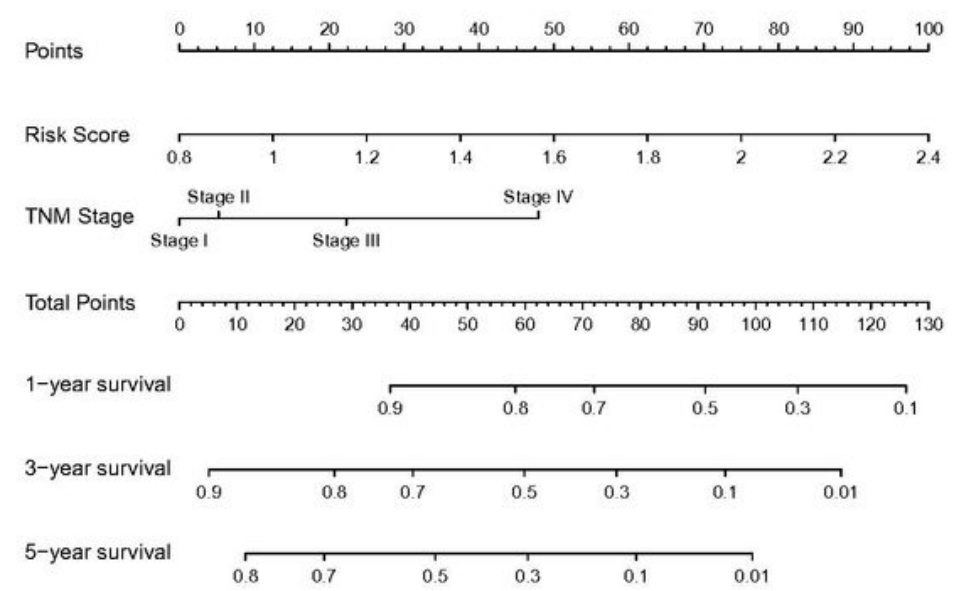

(A)

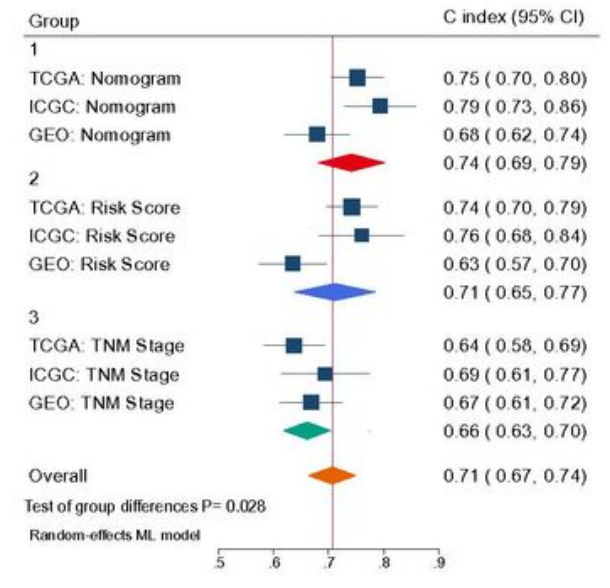

(B)

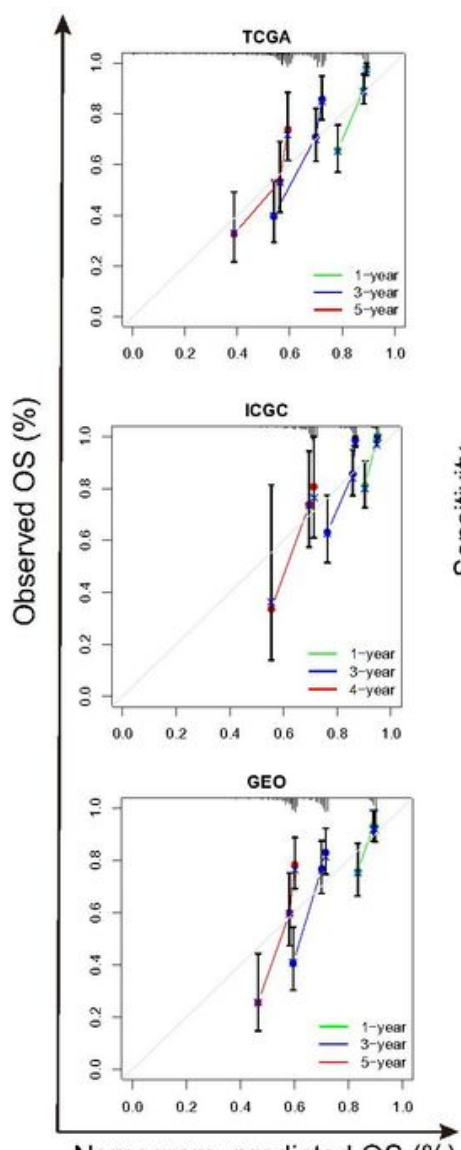

Nomogram-predicted OS (\%)

(C)

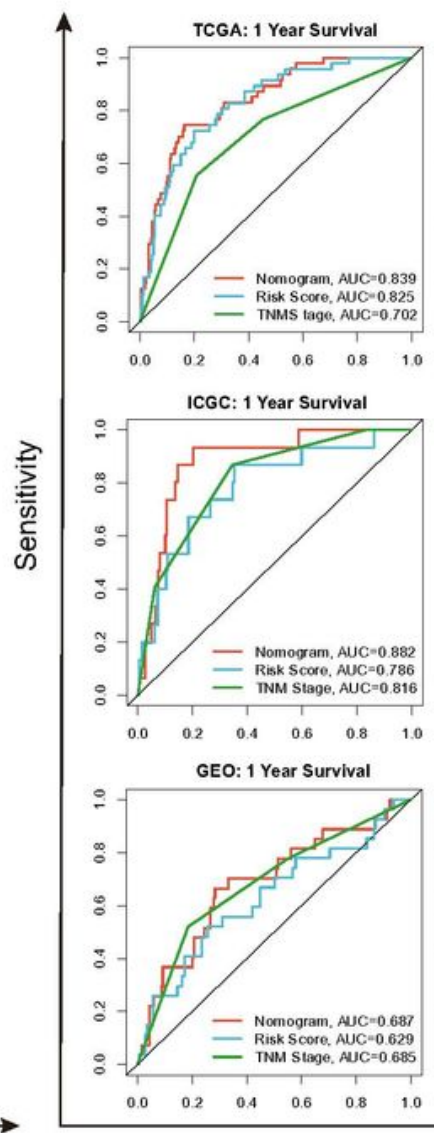

$\begin{array}{llllll}0.0 & 0.2 & 0.4 & 0.6 & 0.8 & 1.0\end{array}$
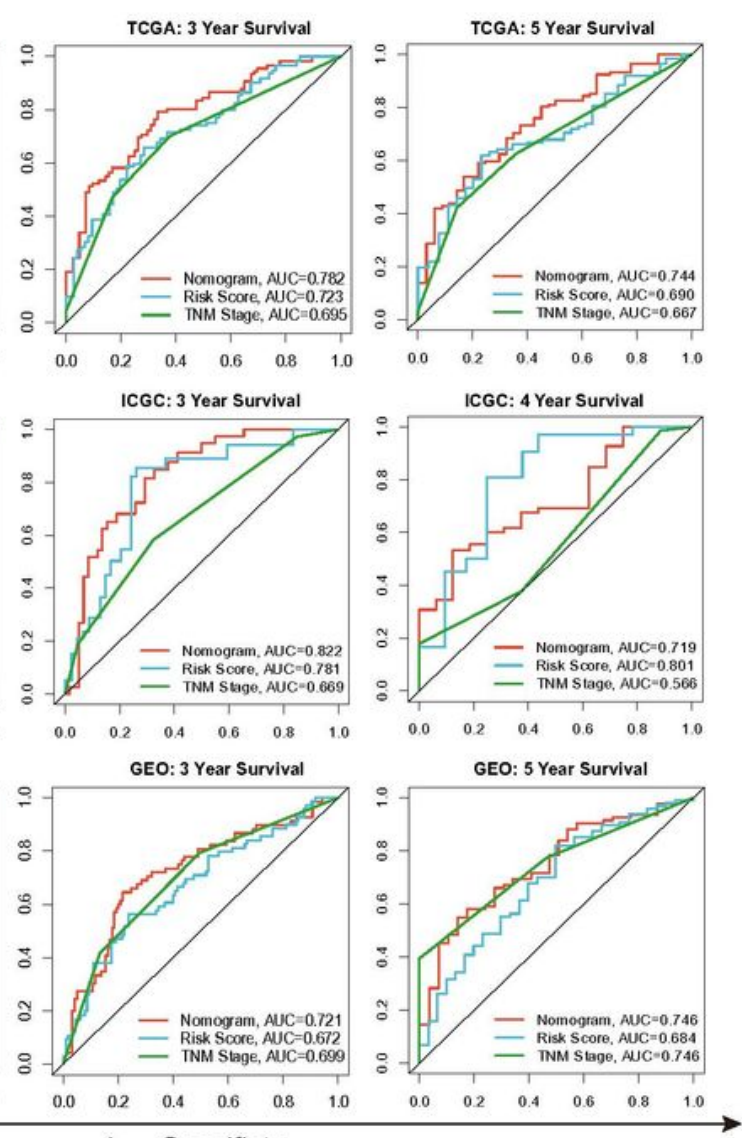

1 - Specificty

(D)

\section{Figure 7}

Development and validation of a predictive nomogram.

(A) The nomogram based on two independent prognostic factors in TCGA-LIHC cohort. (B) The forest plot depicting the pooled concordance indexes of the nomogram, risk score, and TNM stage in TCGA-LIHC, ICGC-LIRI-JP, and GSE1450 cohorts. (C) Calibration plots of the nomogram in TCGA-LIHC, ICGC-LIRI-JP, 
and GSE1450 cohorts. (D) Time-dependent ROC curves showing the comparisons between the nomogram, risk score, and TNM stage in predicting overall survival of TCGA-LIHC, ICGC-LIRI-JP, and GSE1450 cohorts.
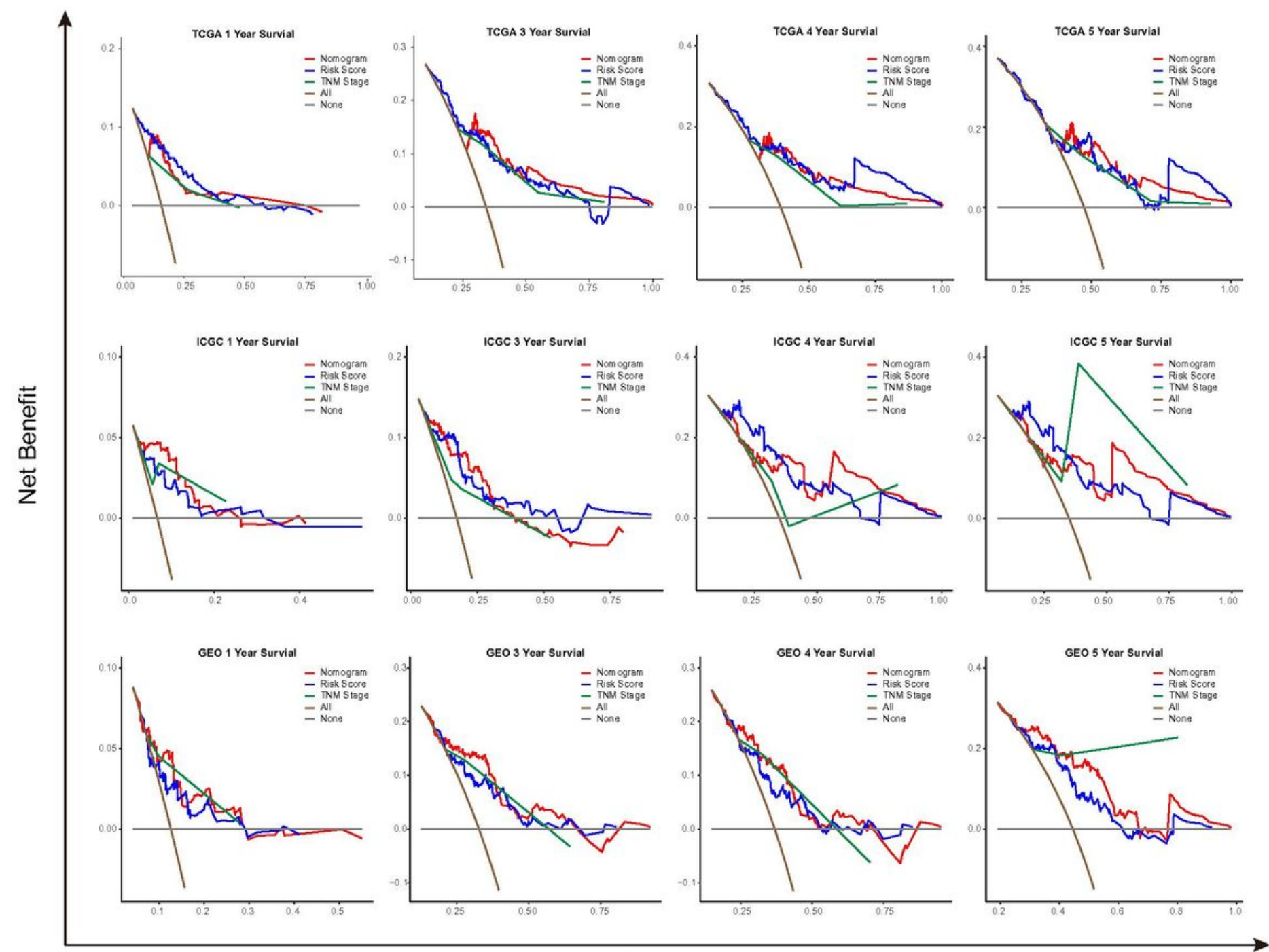

Risk Threshold

\section{Figure 8}

Decision curve analysis (DCA) curves of the nomogram, risk score, and TNM stage. The clinical net benefits were compared in TCGA-LIHC, ICGC-LIRI-JP, and GSE1450 cohorts 


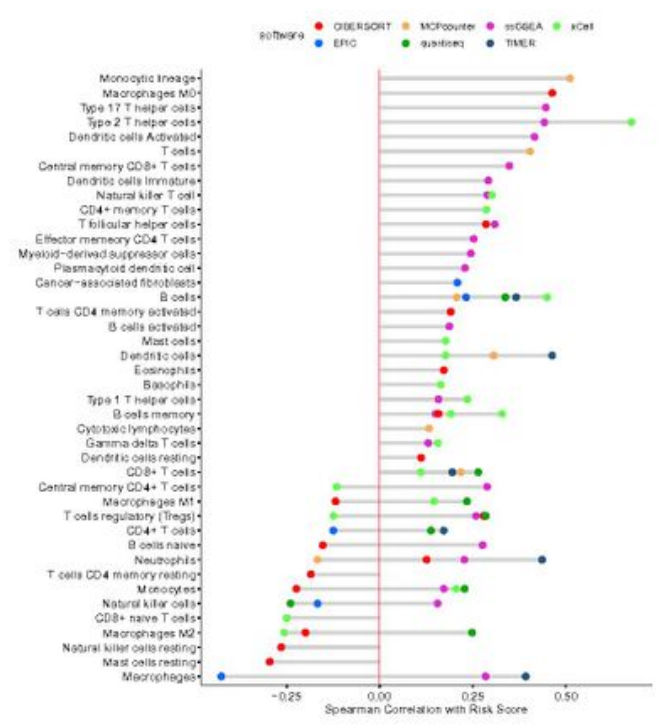

(A)

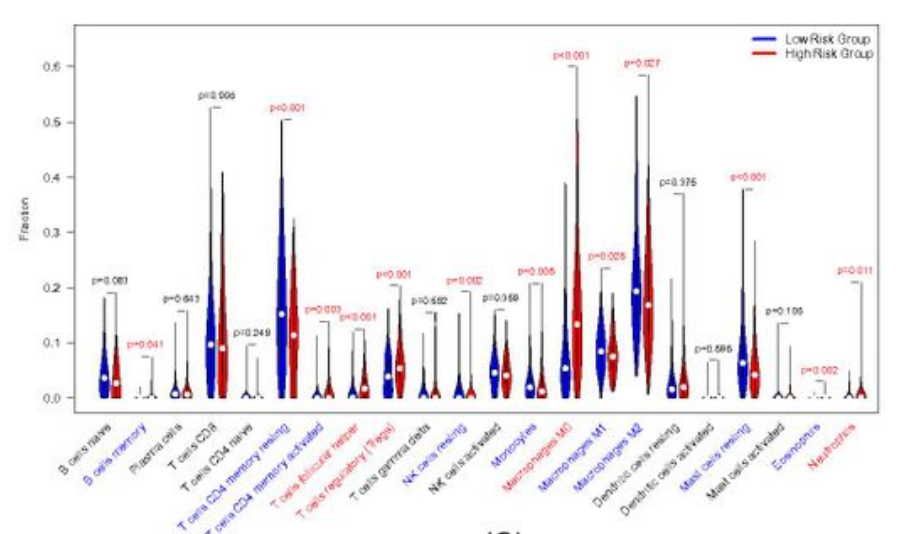

(C)

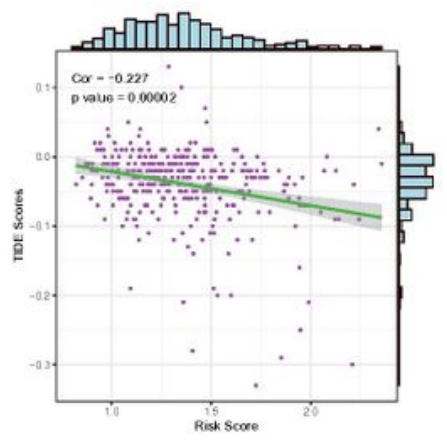

(E)

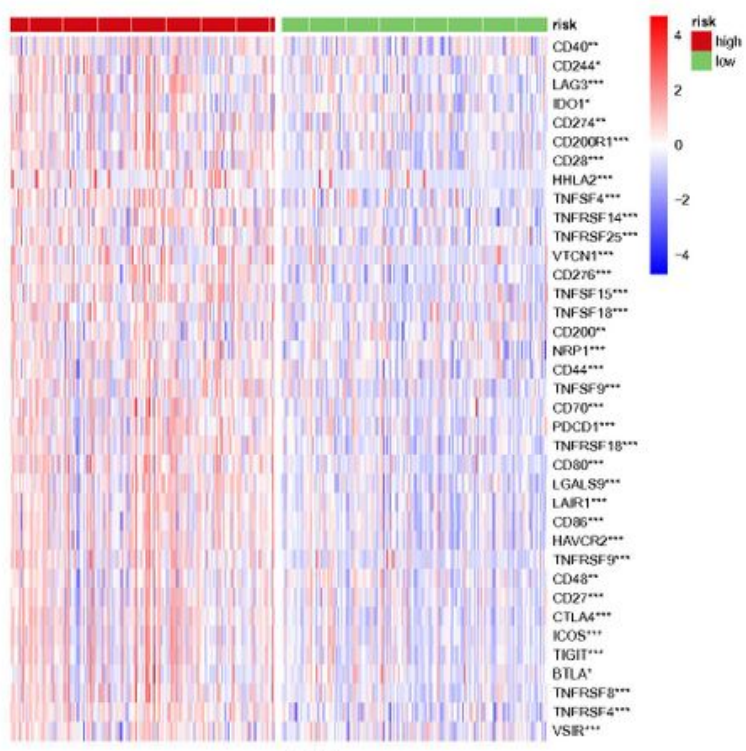

(B)

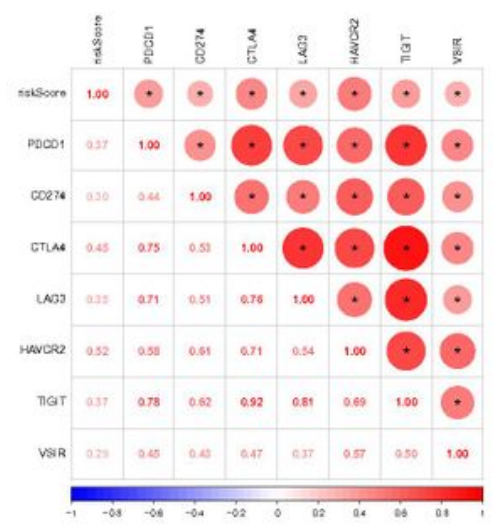

(D)

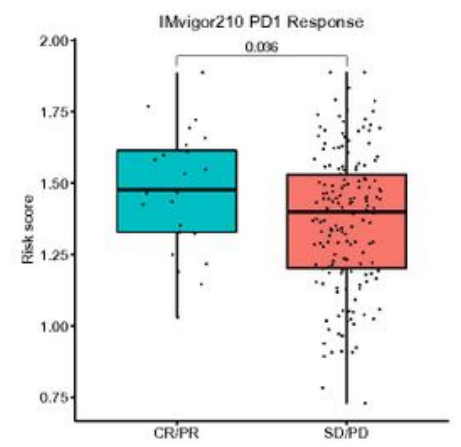

(G)

\section{Figure 9}

Exploration of the tumor microenvironment (TME) and response to immunotherapy in TCGA cohort.

(A) Spearman correlation between the risk score and TME estimated by seven methods. (B) Comparisons of the expressions of immune checkpoint-related genes between high- and low-risk groups (Wilcox test, $* \mathrm{P}<0.05 ; * * \mathrm{P}<0.01 ; * * * \mathrm{P}<0.001 ; * * * * \mathrm{P}<0.0001)$. (C) CIBERORT result of TME between high- and 
low-risk groups (Wilcox test). (D) Spearman correlation analyses between the risk score and seven immune checkpoint-related genes. (E) Spearman correlation between the Tumor Immune Dysfunction and Exclusion (TIDE) score and risk score. (F) Comparison of the TIDE score between high- and low-risk groups (Wilcox test). (G) Comparison of the risk score between responders and non-responders to antiPD1/PD-L1 treatment in the IMvigor210 cohort.
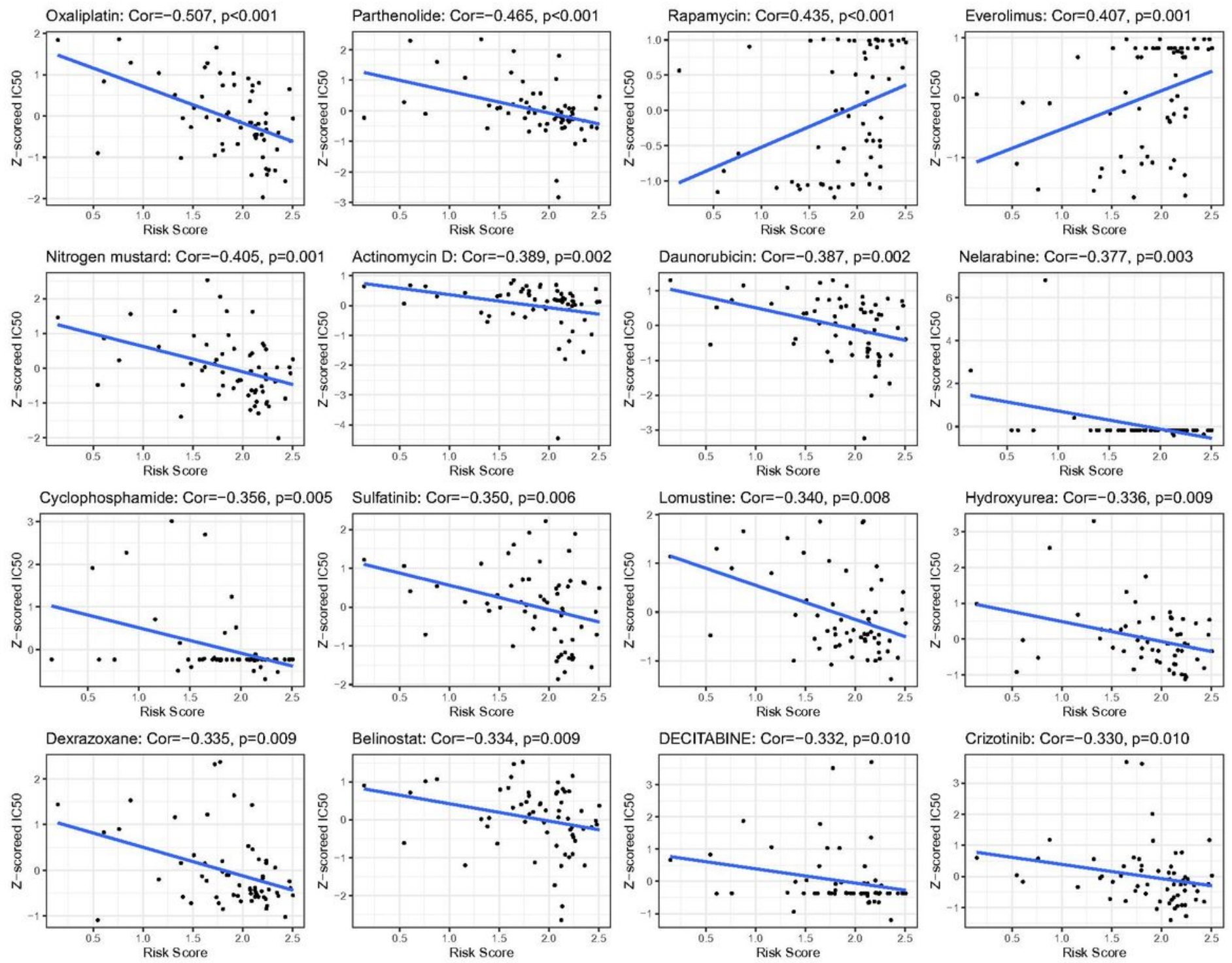

Figure 10

Correlation analyses between drug sensitivity and risk score based on the CellMiner database 

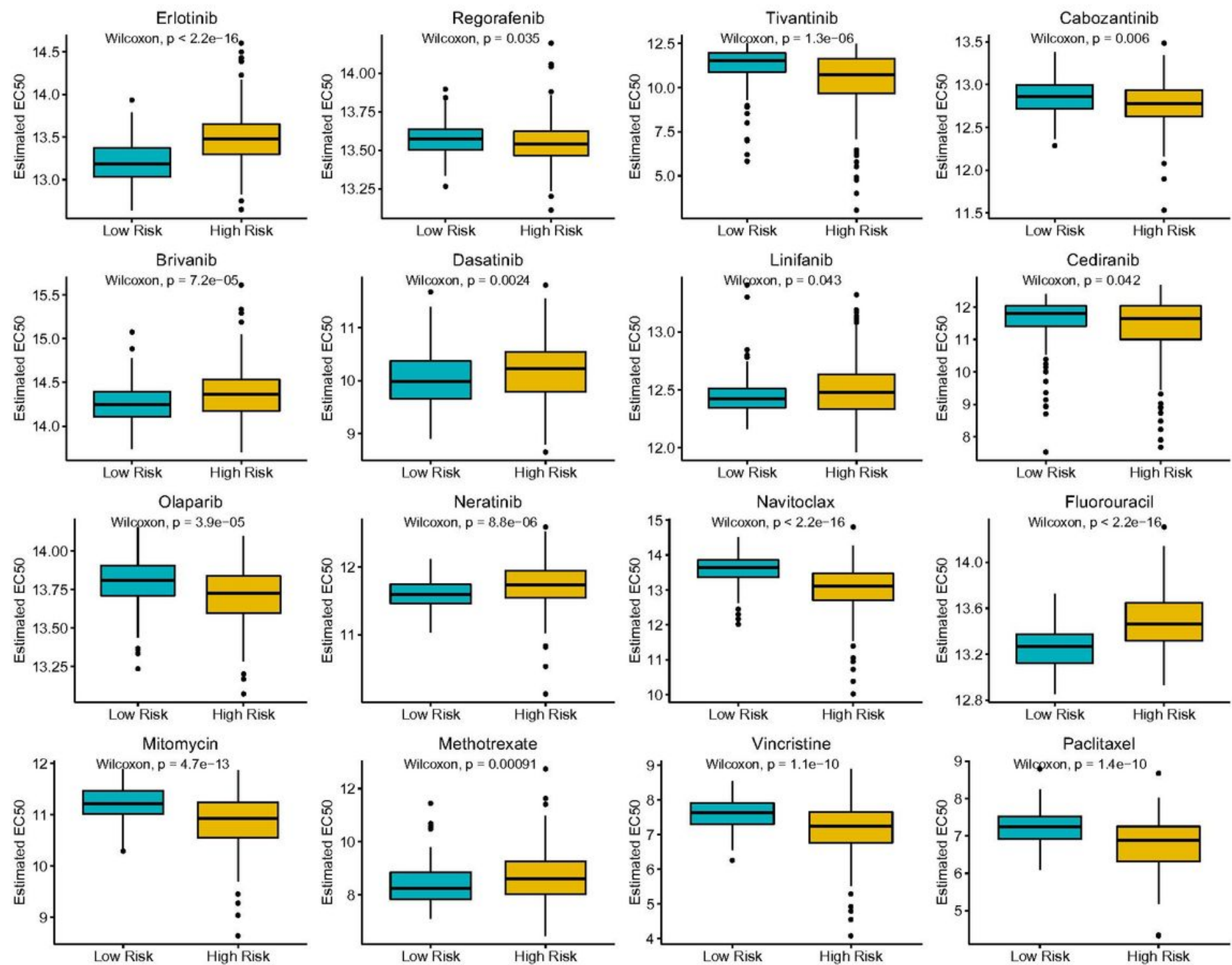

\section{Figure 11}

Prediction of drug sensitivities between high- and low-risk groups in TCGA cohort.

\section{Supplementary Files}

This is a list of supplementary files associated with this preprint. Click to download.

- Additionalfile1Figures1.pdf

- Additionalfile2Figures2.pdf

- Additionalfile3Figures3.pdf

- Additionalfile4Figures4.pdf 
- Additionalfile5TableS1.xlsx

- Additionalfile6Tables2.xIsx

- Additionalfile7TableS3.xIsx

- Additionalfile8TableS4.xlsx

- Additionalfile9TableS5.xlsx 